\title{
Vtc5 is localized to the vacuole membrane by the conserved AP-3 complex to regulate polyphosphate synthesis in budding yeast
}

\author{
Amanda Bentley-DeSousa ${ }^{1,2} \&$ Michael Downey ${ }^{1,2,3}$ \\ 1. Department of Cellular and Molecular Medicine, University of Ottawa, Ottawa, Ontario, \\ Canada \\ 2. Ottawa Institute of Systems Biology, University of Ottawa, Ottawa, Ontario, Canada \\ 3. Corresponding author: mdowne2@uottawa.ca
}

Running Title: AP-3 control of polyphosphate synthesis

Keywords: Polyphosphate, AP-3 complex, Vtc5, polyP, vacuole, Pep4, ESCRT, VTC complex, S. cerevisiae 


\section{HIGHLIGHTS:}

Vtc5 localization to the vacuole membrane depends on the AP-3 complex

The ESCRT pathway brings mislocalized Vtc5 to the vacuole lumen where it is degraded

Decreased polyP levels in AP-3 mutants are explained by Vtc5 mislocalization

Deletion of $D O A 4$ restores wild-type localization of Vtc5 without restoring polyP levels 


\section{ABSTRACT:}

Polyphosphates (polyP) are energy-rich polymers of inorganic phosphates assembled into chains ranging from 3-1000s of residues in length. They are thought to exist in all cells on earth and play roles in an eclectic mix of functions ranging from phosphate homeostasis to cell signaling, infection control, and blood clotting. In the budding yeast Saccharomyces cerevisiae, polyP chains are synthesized by the vacuole-bound VTC complex, which synthesizes polyP while simultaneously translocating it into the vacuole lumen where it is stored at high concentrations. VTC's activity is promoted by an accessory subunit called Vtc5. In this work, we find that the conserved AP-3 complex is required for proper Vtc5 localization to the vacuole membrane. In human cells, previous work has demonstrated that mutation of AP-3 subunits gives rise to Hermansky-Pudlak Syndrome, a rare disease with molecular phenotypes that include decreased polyP accumulation in platelet dense granules. In yeast AP-3 mutants, we find that Vtc5 is rerouted to the vacuole lumen by the ESCRT complex, where it is degraded by the vacuolar protease Pep4. Cells lacking functional AP-3 have decreased levels of polyP, demonstrating that membrane localization of Vtc5 is required for its VTC stimulatory activity in vivo. Our work provides insight into the molecular trafficking of a critical regulator of polyP metabolism in yeast. We speculate that AP-3 may also be responsible for the delivery of polyP regulatory proteins to platelet dense granules in higher eukaryotes. 


\section{INTRODUCTION:}

Polyphosphates (polyP) are chains of inorganic phosphates found in all cell types studied to date. PolyP chains are variable in size, ranging from 3-1000s of units in length, and are linked together via high-energy phosphoanhydride bonds ${ }^{1}$. While once dismissed as 'molecular fossils', recent work suggests that polyP plays critical roles in diverse processes across both prokaryotic and eukaryotic organisms including bacterial virulence, infection control, blood coagulation, protein folding, and diverse aspects of cell signaling ${ }^{2-7}$. As such, polyP chains have gained significant interest as a potential target for therapeutics in a wide variety of pathologies. In contrast to bacteria and fungi, the enzymes that synthesize polyP chains in higher eukaryotic cells are largely unknown. There are no clear homologs of either prokaryotic or fungal polyP synthetases in mammals. Recent work by the Abramov group suggests that the mammalian mitochondrial $\mathrm{F}_{0} \mathrm{~F}_{1}$ ATPase has polyP synthesis capabilities ${ }^{8}$, but the overall contribution of this enzyme to total cellular pools of polyP remains to be tested.

One model organism used to study polyP at a foundational level is the budding yeast $S$. cerevisiae. Here, polyP is present in high concentrations $(>200 \mathrm{mM})$, and the enzymes responsible for its metabolism have been identified ${ }^{9-11}$. In yeast, polyP is synthesized by the vacuole transporter chaperone (VTC) complex. The minimal (core) VTC complex is composed of 3 subunits: Vtc1, Vtc2 or Vtc3, and Vtc4 (the catalytic enzyme) ${ }^{9,12}$. The Vtc3 subunit mostly localizes to the vacuole membrane alongside Vtc1 and $\mathrm{Vtc}^{12}$. On the other hand, the Vtc2 subunit is found at the endoplasmic reticulum and/or cell periphery, and relocalizes to the vacuole membrane under phosphate-limited conditions ${ }^{12}$. PolyP synthesis by VTC requires simultaneous translocation of polyP into the vacuole lumen ${ }^{13}$, where it makes up over $10 \%$ of the dry weight of the cell ${ }^{10}$. PolyP is also found in lower concentrations in the cytoplasm, plasma 
membrane, mitochondria, and nucleus, although observed concentrations vary by method of analysis ${ }^{14}$. In addition to being the only known polyP synthetase in yeast, the VTC complex has been demonstrated or suggested to play a role in a myriad of cellular activities such as the stability of vacuolar V-ATPase subunits, in vacuole fusion, and in microautophagy ${ }^{15-17}$. PolyP itself has also been implicated in the regulation of $\mathrm{pH}$ balance ${ }^{18}$, ion homeostasis ${ }^{19-21}$, and phosphate metabolism ${ }^{18,22-24}$.

Recent work has identified several exciting aspects of VTC regulation. First, activity of the complex is promoted by binding of inositol pyrophosphate InsP7 to the SPX domains of VTC proteins ${ }^{25,26}$. As such, loss of Kcs1, which catalyzes the formation of InsP7, drastically reduces polyP levels ${ }^{27,28}$. Although canonical (serine/threonine) phosphorylation and lysine ubiquitylation sites have been identified on multiple VTC subunits ${ }^{29-31}$, their functions are currently unknown. Recently, a new VTC regulatory subunit termed Vtc5 was identified ${ }^{22}$. Vtc5 localizes exclusively to the vacuole membrane and interacts with the VTC complex to increase the rate of polyP production ${ }^{22}$. PolyP levels in $v t c 5 \Delta$ mutants are reduced to $20 \%$ of those of wild-type cells ${ }^{22}$. The mechanism by which Vtc5 exerts its positive effects on VTC is unknown, although it appears to function independently of effects imparted by inositol pyrophosphates ${ }^{22}$. Given the importance of Vtc5 as a regulator of the core VTC complex, we sought to identify the pathways that are responsible for localizing Vtc5 to the vacuole membrane.

Membrane proteins are synthesized by ribosomes at the endoplasmic reticulum and transported to the trans-golgi network (TGN) prior to being sorted to their final destination ${ }^{32}$. Vacuolar proteins are sorted by two well established protein transport pathways, the CPY

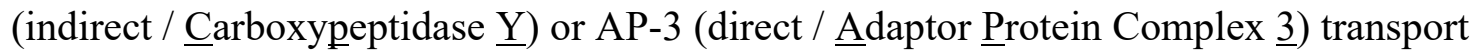
pathways. The CPY pathway transports cargoes to the vacuole in an indirect fashion using the 
endosomal system as an intermediate path. In this pathway, cargoes localize to endosomes prior to fusion with the vacuole membrane for delivery (Fig. 1A $)^{32}$. In contrast, AP-3 cargoes are selected at the TGN, where the complex buds from the TGN with its cargoes to create AP-3 coated vesicles. These vesicles are transported directly to the vacuole through the cytoplasm, and AP-3 docks at the vacuole membrane to release its cargoes (Fig. 1A $)^{32}$. There are links between the AP-3 complex and polyP storage in higher eukaryotes. In humans, mutations in AP-3 are associated with a rare disorder called Hermansky-Pudlak Syndrome ${ }^{33-35}$. Hermansky-Pudlak Syndrome patients lack the ability to synthesize lysosome-related organelles in diverse cell types throughout the body, which results in broad phenotypes that include albinism, visual impairment, and bleeding problems ${ }^{35}$. Notably, this includes the inability to generate dense granules in platelets, where polyP is usually stored in its highest concentrations $(130 \mathrm{mM})^{36}$. PolyP promotes blood clotting by acting on proteins involved in the coagulation cascade including factor XII ${ }^{37}$, factor $\mathrm{XI} / \mathrm{V}^{38,39}$, and thrombin ${ }^{40}$. PolyP addition re-establishes clotting stimulatory activity of platelets derived from Hermansky-Pudlak patients ${ }^{41}$.

In this study, we describe a role for the yeast AP-3 complex in localizing Vtc5 to the vacuole membrane. In AP-3 mutants, Vtc5 is rerouted to the vacuole lumen by the Endosomal

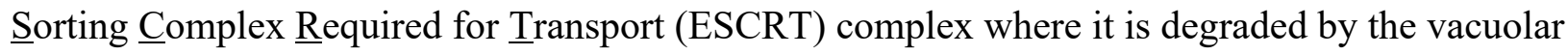
protease Pep4. Mislocalization of Vtc5 in AP-3 mutants is accompanied by a decrease in VTC protein levels and decreased levels of polyP. Overall, our study explains the polyP accumulation defects in AP-3 mutants and provides novel insights into the regulation of the VTC polyP synthetase in budding yeast. 


\section{RESULTS:}

\section{Vtc5 localization to the vacuole membrane is disrupted in AP-3 mutants:}

The CPY and AP-3 pathways comprise the two major routes of protein transport to the vacuole from the TGN (Fig. 1A). To test which of these pathways is responsible for Vtc5 localization to the vacuole membrane, we used live-cell fluorescence microscopy to analyze GFP-Vtc5 localization in AP-3 and CPY pathway mutants. Notably, all N-terminal GFP-Vtc fusions used in this work are functional, with the GFP tag facing the cytoplasmic side of the vacuole membrane to ensure the tag is not degraded within the vacuole lumen ${ }^{12,22}$. These GFP fusions are expressed from constitutive promoters integrated at endogenous VTC loci (See Experimental Procedures). In wild-type cells, GFP-Vtc5 (green) localized exclusively to the vacuole membrane as demonstrated by colocalization with FM4-64 (magenta), a dye which labels the vacuole membrane (Fig. 1B). CPY (pep12A) mutants showed GFP-Vtc5 localization similar to that of wild-type cells (Fig. 1B).

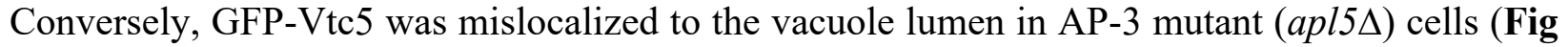
1B). AP-3 is highly conserved from yeast to humans. It consists of a heterotetramer of four protein subunits: two large subunits (Ap15/AP3 $\beta 1$ and Ap16/AP381), one medium subunit (Apm3/AP3 $\mu 1$ ), and one small subunit (Aps3/AP3 $\sigma 1)$ (Supplemental Fig. 1A $)^{42}$. Deletion of genes encoding any one of the four AP-3 subunits results in defects in AP-3 cargo transport ${ }^{43}$. Therefore, to corroborate our results with apl5s, we examined GFP-Vtc5 localization in aps $3 \Delta$ cells and again observed its mislocalization to the vacuole lumen in this mutant (Fig. 1C). Overall, these data suggest that the primary pathway responsible for localizing GFP-Vtc5 to the vacuole membrane is the AP-3 transport pathway, with little if any compensation by the CPY pathway.

Intriguingly, polyP chains can be covalently attached to protein targets as a posttranslational modification termed polyphosphorylation ${ }^{27,44}$. Polyphosphorylation is the non- 
enzymatic addition of polyP chains onto lysine residues, principally within poly-acidic, serine, and lysine (PASK) rich clusters ${ }^{27,45}$. We previously reported that Ap15 is polyphosphorylated in its C-terminus PASK cluster (amino acids 897-932) ${ }^{46}$. Therefore, to test if polyphosphorylation impacts Ap15's role in localizing GFP-Vtc5 to the vacuole membrane, we first deleted the PASK

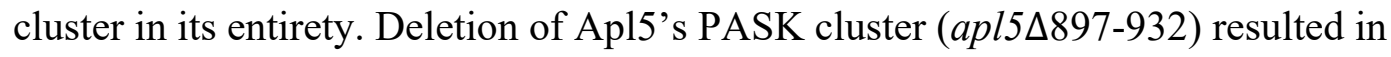
mislocalization of GFP-Vtc5 to the vacuole lumen, similar to what we observed in AP-3 null mutants (Fig. 1C). To define the contribution of polyphosphorylation more specifically, we generated a strain wherein Apl5 is expressed with 13 lysine to arginine (K-R) amino acid substitutions in its PASK cluster. Polyphosphorylation results in an electrophoretic shift of target proteins separated on Bis-Tris NuPAGE gels ${ }^{27,47}$, and this is currently the only method described for evaluation of this new modification. As demonstrated by NuPAGE analysis, the resulting

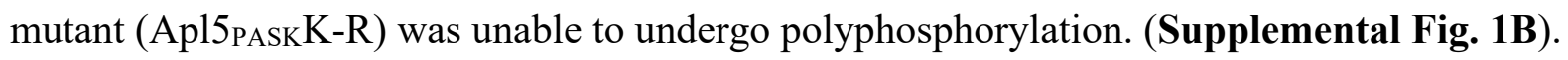
However, this did not impact GFP-Vtc5 localization (Supplemental Fig. 1C), nor did it mimic other known apl5 $\Delta$ phenotypes such as defects in the maturation of AP-3 target vacuolar alkaline phosphatase $\mathrm{Pho}^{48}$ (Supplemental Fig. 1D), or enhanced sensitivity to nickel chloride ${ }^{49}$ or rapamycin $^{50}$ (Supplemental Fig. 2A-B). We note that the PASK cluster lies within Apl5's Vps41 binding domain. Vps41 is a member of the Homotypic Fusion and Protein $\underline{\text { Sorting }}$ (HOPS) complex required for vesicle docking at the vacuole and delivery of AP-3 cargoes ${ }^{51,52}$. As such, the role of the Ap15 PASK in GFP-Vtc5 delivery may stem from disruption of this interaction rather than a defect in polyphosphorylation. Interestingly, the Ap15 C-terminal PASK deletion mutant (apl5 $\Delta 897-932$ ) showed defects in maturation of Pho8, but it did not result in sensitivity to nickel chloride or rapamycin (Supplemental Figs. 1D, 2A-B). This separation of 
function mutant may serve as a useful tool to dissect AP-3's role underlying these distinct phenotypes.

We next sought to test whether vacuolar localization of additional VTC subunits was also impacted by APL5 deletion. We observed no difference in the localization of GFP-Vtc3 in wildtype versus apl5 $\Delta$ mutants (Fig. 2A). GFP-Vtc4 showed an intermediate phenotype, with increased localization to the cytoplasm in $\operatorname{apl} 5 \Delta$, with some protein also remaining at the vacuole membrane (Fig. 2B). These data suggest that although the AP-3 pathway is responsible for the proper vacuolar localization of GFP-Vtc5, other pathways may contribute to the localization of core VTC subunits. Given the importance of Vtc5 as a regulator of VTC activity and the clear disruption of its localization in AP-3 mutants, we focused on the transport of this subunit.

\section{GFP-Vtc subunits are degraded in cells lacking functional AP-3:}

We next used western blotting to gain insight into the fate of mislocalized GFP-Vtc5. Relative to wild-type controls, apl5 $\Delta$ and aps $3 \Delta$ cells showed a striking accumulation of free GFP, which is known to be resistant to degradation (Fig. 3A-B $)^{53}$. This was not the result of increased protein expression, as full length GFP-Vtc5 was reduced in these mutants (Fig. 3A \& 3C). This same pattern has been observed previously for other proteins mislocalized to the vacuole lumen and is attributed to cargo degradation ${ }^{54,55}$. We also tested if GFP-Vtc4 and GFP-Vtc3 levels were similarly affected. Both GFP-Vtc4 and GFP-Vtc3 also accumulated free GFP at the expense of decreased full-length fusions, although the effect was not as dramatic as that observed for GFPVtc5 (Fig. 3D-3I). Previous work from the Mayer group showed that $v t c 5 \Delta$ cells have decreased levels of core VTC subunits at the vacuole membrane ${ }^{22}$. Degradation of GFP-Vtc3 and GFPVtc4, and partial mislocalization of GFP-Vtc4 in apl5s cells, are consistent with a model wherein mislocalized Vtc5 is largely non-functional in the absence of AP-3. However, we cannot 
exclude that these molecular phenotypes stem in part from defects in the transport of other AP-3 cargos (see Discussion).

\section{Mislocalized Vtc5 is rerouted to the vacuole lumen by the ESCRT pathway:}

We next investigated which pathways are responsible for localizing GFP-Vtc5 to the vacuole lumen in the absence of functional AP-3, with a focus on the autophagy and ESCRT pathways. Autophagy is a process whereby cytoplasmic material becomes sequestered in vesicles that fuse to the vacuole to deliver their contents for degradation ${ }^{56}$. The ESCRT pathway is responsible for detecting ubiquitylated transmembrane proteins to sort them into multi-vesicular bodies (MVBs) in the endocytic pathway for delivery to the vacuole ${ }^{57}$. Disruption of ESCRT ( vps274), but not autophagy $(\operatorname{atg} 8 \Delta)$ in AP-3 mutants resulted in a loss of GFP signal from the vacuole lumen (Fig. 4A). In contrast, neither pathway impacted GFP-Vtc5 localization in wild-type cells (Supplemental Fig. 3A). When ESCRT complex subunits are mutated, the complex becomes defective for proper MVB biogenesis and fusion at the vacuole membrane ${ }^{57}$. Indeed, in apl5s vps $27 \Delta$ double mutants, GFP-Vtc5 appeared to accumulate in FM4-64 labelled MVBs around the vacuole membrane (Fig. 4A). Deletion of VPS27 also resulted in reversal of free GFP accumulation in western blots (Fig. 4B). This same molecular phenotype was observed across multiple ESCRT complex mutants (ESCRT-0, -I, -II, and -III, Supplemental Fig. 3B).

The ESCRT pathway recognizes cargoes that have been ubiquitylated by E3 ubiquitin $\operatorname{ligases}^{57,58}$. These cargoes are then transported to the vacuole via MVBs that fuse with the vacuole membrane to deliver its contents. As one of the last steps in this process, the deubiquitinase Doa4 removes ubiquitin moieties from ESCRT-targeted proteins in order to recycle ubiquitin levels within the cell upon cargo delivery to the vacuole ${ }^{57,58}$. Mutation of DOA4 alone has no impact on GFP-Vtc5 processing or localization (Fig. 4C and Supplemental 
Fig. 3C). In AP-3 mutants, however, doa4d prevented the accumulation free GFP, mirroring what was seen for ESCRT mutants (Fig. 4C). However, in contrast to the ESCRT mutants, doa4 $\Delta$ also restored robust GFP-Vtc5 localization to the vacuole membrane (Fig. 4D).

Altogether, we conclude that Doa4 is a key player in localizing Vtc5 to the vacuole lumen in the absence of AP-3. Finally, when proteins accumulate in the lumen, they become accessible to vacuole proteases $^{59}$. Deletion of the major vacuole protease Pep4 largely rescued both full-length GFP-Vtc5 and reversed the accumulation of free GFP in AP-3 mutants (Fig. 4A-B), suggesting that Pep4 is a major protease responsible for Vtc5 degradation.

\section{The AP-3 complex is required for maintenance of wild-type polyP levels:}

Finally, we tested the contribution of AP-3 to polyP homeostasis. Deletion of AP-3 subunits resulted in a clear decrease in polyP levels (Fig. 5A). This finding is consistent with previous data wherein AP-3 mutants were found to be important for polyP accumulation in a large scale screen $^{60}$. We observed a similar result in our strains used for prior analyses where GFP-Vtc5 is expressed under a constitutive GPD1 promoter (Fig. 5B). Together with our previous results, these data suggest that correct localization of Vtc5 to the vacuole membrane by the AP-3 complex is important for its function. Notably, AP-3 mutants still have more polyP than cells lacking Vtc5 altogether (vtc5s, Fig. 5C). This observation could be explained by residual localization of Vtc5 to the vacuole membrane in AP-3 mutants. Interestingly, deletion of DOA4, which rescues GFP-Vtc5 protein levels and localization to the vacuole membrane, was unable to reverse the decreased polyP levels in apl5s mutants (Fig. 5D). Thus, proper function of GFPVtc5 requires localization to the vacuole membrane, specifically through the AP-3 pathway. 


\section{DISCUSSION:}

\section{AP-3 regulation of $\mathrm{Vtc5}$ localization:}

Interest in polyP research has experienced a resurgence in recent years on the heels of exciting connections between polyP and diverse aspects of cell signaling and protein homeostasis. With the key players involved in mammalian polyP metabolism largely uncharacterized, model systems have proved essential to our understanding of polyP dynamics and function. In $S$. cerevisiae, polyP is synthesized by the vacuole-bound VTC complex and stored at high concentrations in the vacuole ${ }^{13}$. The activity of the core VTC complex (consisting of Vtc1, Vtc2 or Vtc3, and Vtc4) is increased dramatically by the Vtc5 subunit ${ }^{22}$. This is the only protein known to act directly on the VTC complex to stimulate polyP production. Our study supports a model where Vtc5 localization to the vacuole membrane depends on the evolutionarily conserved AP-3 complex. This finding provides insight into the regulation of the VTC complex and identifies Vtc5 as a cargo whose mislocalization underlies polyP accumulation defects observed in AP-3 mutants.

In wild-type cells (Fig. 6), we propose that Vtc5 is sorted into AP-3 coated vesicles at the TGN and transported directly to the vacuole. The AP-3 complex selects protein cargoes by tyrosine-based motifs (YXXØ where $\mathrm{X}$ represents any amino acid and $\varnothing$ is a bulky hydrophobic amino acid) and/or dileucine-based motifs ([D/E]XXXL[L/I] where X represents any amino acid $)^{61}$. Notably, Vtc5 has 6 tyrosine-based motifs and 2 dileucine-based motifs which together could be used as a targeting signal. Alternatively, Vtc5 could be transported in conjunction with other AP-3 cargoes such as Vam3, which is also required for wild-type levels of polyP accumulation $^{60}$. Regardless, we propose that AP-3 coated vesicles may bind to Vps41 of the HOPS complex to facilitate docking and release of cargo, including Vtc5, into the vacuole 
membrane. In the absence of AP-3, Vtc5 is mislocalized to the vacuole lumen. Mislocalized

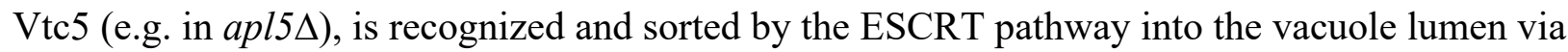
the endosomal system where it is eventually degraded in a manner dependent on vacuole protease Pep4. Since Pep4 is also required for the activation of vacuole proteases Prb1 and $\operatorname{Prc1}{ }^{62,63}$, it is possible that these also these play a role in Vtc5 degradation in the vacuole lumen. We found that degradation of Vtc5 in AP-3 mutants requires the Doa4 deubiquitinase. The involvement of Doa4 suggests that mislocalized Vtc5 is subject to regulation by ubiquitylation. In support of this assertion, several large-scale studies have identified ubiquitylation sites in the middle and C-terminal regions of the protein ${ }^{64}$. We speculate that these sites may be targets of the Rsp5 E3 ubiquitin ligase that has been implicated in the ESCRTdependent delivery of proteins to the vacuole lumen ${ }^{65-67}$. Notably, Vtc5 is also rich in phosphoserines targeted by both the Cdk1 (cell cycle) and Nkk1(nitrogen metabolism) ${ }^{64}$. These modifications could modulate Vtc5 localization in wild-type or AP-3 mutant cells or may instead be involved in regulating its activity towards the core VTC complex.

\section{Regulation of the core VTC complex:}

In contrast to GFP-Vtc5, we found that localization of GFP-Vtc3 to the vacuole membrane was not impacted by disruption of AP-3 and GFP-Vtc4 was only partially affected. However, deletion of AP-3 subunits still reduced GFP-Vtc3/4 protein levels and the increased appearance of free GFP in western blots, albeit to a lesser degree than that observed for GFP-Vtc5. We suggest that transport of core VTC subunits occurs through multiple transport routes, although these may function redundantly with AP-3. This type of dual regulation has been documented for other AP-3 cargos such as Sna $4^{55}$ and Ypq1 ${ }^{68}$. It is also possible that changes observed in core subunits stem from mislocalized Vtc5. There is strong evidence that maximal polyP production 
requires VTC localization to the vacuole. However, polyP has also been detected in variable amounts at the plasma membrane, cytoplasm, mitochondria, and nucleus ${ }^{14}$. Whether vacuolar polyP is somehow transported to these compartments or if it is made by VTC residing locally remains an open question. In the latter case, it is possible that Vtc5 association (or lack thereof) with core VTC in these areas also determines subcellular concentrations of polyP outside of the vacuole. In future work it will be intriguing to identify pathways that regulate localization of GFP-Vtc2, which is found at the plasma membrane or peripheral endoplasmic reticulum in phosphate replete conditions, becoming enriched at the vacuole only under conditions of phosphate starvation $^{9,12,15}$.

\section{$\underline{\text { AP-3 impact on polyP levels: }}$}

Our work shows that AP-3 mutant cells have reduced levels of polyP. This was true in both a wild-type background and under conditions of Vtc5 overexpression. These findings are consistent with work from Feimoser et al. identifying AP-3-encoding genes in a genome-wide screen for deletion mutations with defects in polyP accumulation ${ }^{60}$. Interestingly, deletion of DOA4 in an AP-3 mutant background rescued both GFP-Vtc5's localization to the vacuole membrane and restored wild-type levels of full-length GFP-Vtc5 protein without restoring polyP levels. In fact, polyP levels in apl5 $\Delta$ doa $4 \Delta$ double mutants were lower than in either single mutant. While this may seem counterintuitive, we suggest that Vtc5 is not correctly positioned within the vacuole membrane under these circumstances and unable to stimulate VTC activity.

The reduction in polyP levels seen in AP-3 is not as dramatic as that observed in cells lacking Vtc5 altogether, suggesting either residual localization of Vtc5 to the vacuole membrane in AP-3 mutants, or that mislocalized Vtc5 remains competent to stimulate core VTC activity to some degree. Notably, Freimoser et al. identified over 200 additional genes that impact polyP 
metabolism ${ }^{60}$, and some of these could function as direct regulators of Vtc5 or AP-3.

We recently described the Ap15 subunit of AP-3 as a target of lysine

polyphoshorylation $^{46}$. However, our analysis of mutant Ap15 that cannot be polyphosphorylated suggests that this modification does not impact GFP-Vtc5 delivery to the vacuole or other AP-3 related phenotypes that we tested. Polyphosphorylation of AP-3 may become important under select stress conditions that remain to be identified. Since polyP has been described as having chaperone activity ${ }^{4}$, another possibility is that polyphosphorylation promotes degradation or refolding of a small fraction of AP-3 that is itself mislocalized to the polyP-rich vacuole lumen.

\section{Conservation of the AP-3 complex and function:}

The AP-3 complex is highly conserved in mammalian cells in terms of subunit organization and function. For example, the human homolog of Ypq1, PQLC2, is transported to the vacuole membrane by the AP-3 complex when it is expressed in yeast ${ }^{68}$. Notably, mutations in human AP-3 give rise to Hermansky-Pudlak Syndrome. Relevant here is the observation that molecular phenotypes of Hermansky-Pudlak Syndrome patients include a failure to accumulate polyP in platelet dense granules, a type of lysosome-related organelle conceptually similar to the yeast vacuole. Based on the conserved role of AP-3 subunits in polyP metabolism, we suggest that identification and characterization of AP-3 cargoes in human cells that accumulate high levels of polyP may provide unique insights into the human polyP synthetases, the identity of which remains a critical open question in the field. 


\section{EXPERIMENTAL PROCEDURES:}

Yeast strains \& handling: Yeast strains were constructed using standard techniques via transformation of PCR products containing selectable markers. For gene deletions, PCR analyses were used to confirm the position of gene deletion and the absence of wild-type gene copy. PCR was also used to confirm the correct genomic location of cassettes used for epitope tagging. Genotypes for all strains used in this study are listed in Table S1. For all experiments performed strains were grown in YPD (2\% glucose supplemented with $0.005 \%$ Adenine and $0.005 \%$ Tryptophan).

Apl5 K-R Mutagenesis: A construct containing the lysine $(\mathrm{K})$ to arginine $(\mathrm{R})$ mutations within Ap15 PASK cluster was created custom by GenScript and used for the experiments listed in Supplemental Fig 1 and Supplemental Fig 2. The construct was integrated with a GFP tag or flag tag at the endogenous APL5 locus using transformation of overlapping PCR products.

Electrophoresis \& Immunoblotting: Methods for protein extraction were described previously ${ }^{47}$ and are summarized here using similar wording for clarity. A BioSpec bead-beater was used to lyse cell pellets corresponding to 3-6 OD 600 units in the presence of $100 \mu \mathrm{L}$ of acid washed beads and $300 \mu \mathrm{L}$ of $20 \%$ TCA (Sigma-Aldrich T6399). 2 x 3-minute pulses were used. The supernatant was recovered, and cells were washed with $300 \mu \mathrm{L}$ of $5 \%$ TCA (Sigma-Aldrich T6399). The supernatant from the second wash was combined with the first and this mixture was clarified by centrifugation at $4{ }^{\circ} \mathrm{C}$ at $16,000 \times g$ for 4 minutes. The supernatant was removed, and the pellet was resuspended in SDS-PAGE sample buffer (see buffer recipes section) supplemented with 1/10 volume of 1.5M Tris-HCl pH 8.8 (Tris Base Fisher BP152-5, Hydrochloric Acid Fisher A144-212) and 1/10 volume 1 M DTT (Bio Basic DB0058). Samples were boiled for 5 minutes before an additional centrifugation at $4{ }^{\circ} \mathrm{C}$ at $16,000 \mathrm{x} g$ for 4 minutes. 
The supernatant was recovered and stored or used immediately for SDS-PAGE or NuPAGE analysis (Thermo Fisher NP0336). Where indicated, BioRad TGX Stain-Free ${ }^{\mathrm{TM}}$ FastCast $^{\mathrm{TM}} 10 \%$ acrylamide (BioRad 1610183) was used for quantification with total protein quantified in place of a loading control. BioRad TGX acrylamide gels were transferred to nitrocellulose membrane (BioRad 162-0112) and exposures were obtained using a Bio-Rad ChemiDoc system. Non-TGX 12\% SDS-PAGE and NuPAGE gels (Thermo Fisher NP0336) were transferred to PVDF membrane (BioRad 162-0177) and exposures were obtained using autoradiography film (Harvard Apparatus Canada DV-E3018). In all cases, Chemiluminescence Luminata Forte ECL (Fisher Scientific WBLUF0500) was employed for detection. All antibodies used for immunoblotting are described in Table S2.

Spot tests: Cells were diluted to $0.1 \mathrm{OD}_{600}$, grown for 4 hours, then diluted to $0.1 \mathrm{OD}_{600}$ prior to being serially diluted 5-fold in $\mathrm{H}_{2} 0.4 \mu \mathrm{L}$ of each dilution was spotted on the indicated media prior to incubation at $30{ }^{\circ} \mathrm{C}$ for 2-3 days. Plates contained the following chemicals/drugs: 0.028\% DMSO (VWR CA97061-250), $0.75 \mathrm{mM} \mathrm{NiCl}_{2}$ (Fisher N54-250), and $0.003 \mu \mathrm{M}$ Rapamycin (Sigma-Aldrich R0395-1MG). Images were taken using a Bio-Rad ChemiDoc. Microscopy: Live-cell fluorescence imaging was conducted using a Leica DMI 6000 with a Hamamatsu camera using the Volocity® 4.3.2 imaging program. Briefly, cells were diluted to $\mathrm{OD}_{600}=0.2$ from overnight cultures in YPD. After 3 hours of growth at $30{ }^{\circ} \mathrm{C}, \mathrm{FM} 4-64$ (Thermo Fisher T13320; 1.64 mM stock in DMSO) was added to a final concentration of 1.64 $\mu \mathrm{M}$ for an additional 2 hours. Cells were then washed out in YPD for 30 minutes at room temperature. In instances where there was a difference in protein level expression, exposure times were taken at longer intervals to account for this discrepancy (Fig. 1). For microscopy in Fig. 2, images were taken on a Zeiss AxioObserver 7 with a Hamamatsu ORCA-Flash LT 
camera using Zeiss Zen 3.0 Pro Software. All images were taken with oil immersion at 63X. All images were then analyzed in FIJI. Backgrounds were subtracted with a rolling ball radius of 50 pixels and images taken with FM4-64 dye were converted from red to magenta.

Polyphosphate Extractions: Polyphosphate was extracted from yeast pellets containing 8-12 $\mathrm{OD}_{600}$ units using an adapted protocol from Bru et al 2016 ${ }^{7,69}$. Cells were resuspended in $400 \mu \mathrm{L}$ of cold LETS buffer (see buffer recipes section). Subsequently, $600 \mu \mathrm{L}$ of neutral phenol $\mathrm{pH} 8$ (Sigma-Aldrich P4557) and $150 \mu \mathrm{L}$ of $\mathrm{mH}_{2} \mathrm{O}$ were added. Samples were vortexed for 20 seconds and heated for 5 minutes at $65^{\circ} \mathrm{C}$ followed by a 1-minute incubation on ice. $600 \mu \mathrm{L}$ of chloroform (Sigma-Aldrich 472476) was added, samples were vortexed for 20 seconds and spun down at room temperature for 2 minutes at $13,000 \mathrm{x}$. The top layer was then transferred to a new tube containing $600 \mu \mathrm{L}$ of chloroform (Sigma-Aldrich 472476), vortexed for 20 seconds and spun down at room temperature for 2 minutes at $13,000 \mathrm{x} g$. The top layer was transferred to a new tube and $2 \mathrm{uL}$ of RNAse A (10 mg/mL; Thermo Fisher R1253) and $2 \mu \mathrm{L}$ of DNAse I (10 $\mathrm{mg} / \mathrm{mL}$; Thermo Fisher AM2222) were added followed by a 1 -hour incubation at $37^{\circ} \mathrm{C}$. The

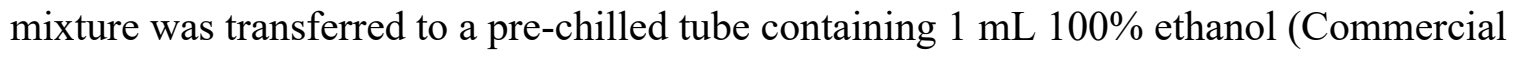
Alcohols P006EAAN) and $40 \mu \mathrm{L}$ of 3M sodium acetate $\mathrm{pH} 5.3$ (Sigma-Aldrich S7899). Samples were left at $-20^{\circ} \mathrm{C}$ overnight and then centrifuged for 20 minutes at $13,000 \times g$ at $4^{\circ} \mathrm{C}$. The pellet was washed in $500 \mu \mathrm{L}$ of cold 70\% ethanol (Commercial Alcohols P006EAAN), centrifuged for 5 minutes at $13,000 \times \mathrm{g}$ at $4^{\circ} \mathrm{C}$ then the supernatant was discarded, and the pellet was dried. The pellet was resuspended in 20-30 $\mu \mathrm{L} \mathrm{mH}_{2} \mathrm{O}$. Samples were mixed 1:1 with polyP loading dye (see buffer recipes section) and electrophoresed on a 15.8\% TBE-Urea Acrylamide Gel at 100V for 1 hour and 45 minutes in $1 \mathrm{X}$ TBE buffer (see buffer recipes section). The gel was incubated in fixing solution (see buffer recipes section) with toluidine blue for 15 minutes and then destained 
in destaining solution. PolyP standards (a gift from T. Shiba) were used to assess polyP chain length.

Statistical analyses: For statistical analyses performed on western blots in Fig. 3, a one-way ANOVA was performed with Tukey posthoc tests at 95\% confidence intervals. Error bars, pvalues and number of biological replicates (n) are defined in the relevant figure legends.

\section{Buffer recipes:}

The following buffer recipes are taken from our previous study (Bentley-DeSousa et al., 2018) and are listed here again verbatim for convenience.

\section{SDS-PAGE Running Buffer (1X working):}

$100 \mathrm{~mL}$ of 10X 1 litre Stock [30.2 g Tris Base (Fisher BP152-5), $188 \mathrm{~g}$ Glycine (Fisher BP3815), $10 \mathrm{~g}$ SDS (Fisher BP166)] and $900 \mathrm{~mL} \mathrm{ddH}_{2} \mathrm{O}$. * Please note SDS-PAGE gels don't resolve polyP shifts*

NuPAGE Running Buffer (1X working):

$50 \mathrm{~mL}$ of 20X 1 litre Stock [209.2 g MOPS (Sigma M1254), 121.1 g Bis-Tris (Sigma B9754), 20 g SDS (Fisher BP166), $12 \mathrm{~g}$ EDTA (Sigma ED2SS)], $5 \mathrm{~mL}$ of 1M Sodium Bisulfate (Fisher

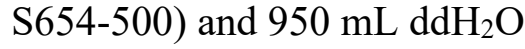

SDS-PAGE Transfer Buffer (1X working):

$100 \mathrm{~mL}$ of 10X 1L Stock [30.275 g Tris Base (Fisher BP152-5), 166.175 Glycine (BioBasic GB0235], $200 \mathrm{~mL}$ Methanol (Fisher A412P-4) and $700 \mathrm{~mL} \mathrm{ddH_{2 } \mathrm { O }}$

\section{NuPAGE Transfer Buffer (1X working):}

$50 \mathrm{~mL}$ of 20X 1L Stock [81.6 g Bicine (Sigma B3876), $104.8 \mathrm{~g}$ Bis-Tris (Sigma B9754), $6 \mathrm{~g}$

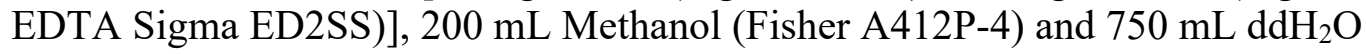

SDS-PAGE/NuPAGE Sample Buffer: (3X stock):

$800 \mu \mathrm{L}$ of stock [160 mM Tris-HCl pH 6.8 (Tris base Fisher BP152-5, Hydrochloric acid Fisher A144-212), 6\% SDS w/v (Fisher BP166), 30\% Glycerol (Fisher BP229-4), 0.004 \%

Bromophenol Blue (Fisher BP115-25)]. For TCA preps, 3X is supplemented with $100 \mu \mathrm{L} 1 \mathrm{M}$ DTT (BioBasic DB0058), and $100 \mu \mathrm{L}$ 1.5M Tris-HCl (pH 8.8) [Tris base (Fisher BP152-5), Hydrochloric acid (Fisher A144-212) 
The following buffer recipes are used in Bentley-DeSousa et al., 2021.

TBE (1X working):

$200 \mathrm{~mL}$ of a $5 \mathrm{X}$ Stock [67.5 g Tris Base (Fisher BP152-5), $34.37 \mathrm{~g}$ Boric Acid (Fisher BP168-1), and $25 \mathrm{~mL}$ 0.5M EDTA (Sigma-Aldrich 03690)]

LETS Buffer (1X working):

100 mM LiCl (Fisher L120-500), 10 mM EDTA (Sigma-Aldrich 03690), 10 mM Tris-HCl pH

7.4 [(Tris Base Fisher BP152-5, Hydrochloric Acid Fisher A144-212)], 20\% SDS (Thermo

Fisher AM9820)

PolyP Loading Dye (6X):

10 mM Tris-HCl pH 7 [(Tris Base Fisher BP152-5, Hydrochloric acid Fisher A144-212)], $1 \mathrm{mM}$ EDTA (Sigma-Aldrich 03690), 30\% Glycerol (Fisher BP229-4), and Bromophenol Blue (Fisher BP115-25)

\section{Toluidine Blue Fixing Solution:}

25\% Methanol (Fisher A412P), 5\% Glycerol (Fisher BP229-4), 0.05\% Toluidine Blue (SigmaAldrich T3260)

Destaining Solution:

25\% Methanol (Fisher A412P), 5\% Glycerol (Fisher BP229-4)

\section{CONFLICTS OF INTERESTS:}

The authors declare no conflicts.

\section{ACKNOWLEDGEMENTS:}

We thank A. Mayer for yeast strains, T. Shiba (Regentiss, Japan) for polyP standards and members of the Downey lab for critical reading of the manuscript. We also acknowledge the Cell Biology and Image Acquisition Core funded by the University of Ottawa, Ottawa, Canada and the Canada Foundation for Innovation for microscopy training and expertise. Work for this project was funded by a Canadian Institutes of Health Research (CIHR) Project Grant (PJT148722), and by an Early Researcher Award from the Ontario Ministry of Innovation and Research to MD. ABD was funded in part by a graduate scholarship from the Natural Sciences and Engineering Research Council of Canada (NSERC). 


\section{REFERENCES:}

1. Rao, N.N., Gomez-Garcia, M.R. \& Kornberg, A. Inorganic polyphosphate: essential for growth and survival. Annu Rev Biochem 78, 605-647 (2009).

2. Roewe, J. et al. Bacterial polyphosphates interfere with the innate host defense to infection. Nat Commun 11, 4035 (2020).

3. Travers, R.J., Smith, S.A. \& Morrissey, J.H. Polyphosphate, platelets, and coagulation. Int J Lab Hematol 37 Suppl 1, 31-35 (2015).

4. Gray, M.J. et al. Polyphosphate is a primordial chaperone. Mol Cell 53, 689-699 (2014).

5. Dahl, J.U. et al. The anti-inflammatory drug mesalamine targets bacterial polyphosphate accumulation. Nat Microbiol 2, 16267 (2017).

6. Xie, L. \& Jakob, U. Inorganic polyphosphate, a multifunctional polyanionic protein scaffold. J Biol Chem 294, 2180-2190 (2019).

7. Bondy-Chorney, E. et al. A Broad Response to Intracellular Long-Chain Polyphosphate in Human Cells. Cell Rep 33, 108318 (2020).

8. Baev, A.Y., Angelova, P.R. \& Abramov, A.Y. Inorganic polyphosphate is produced and hydrolyzed in F0F1-ATP synthase of mammalian mitochondria. Biochem J 477, 1515 1524 (2020).

9. Gerasimaite, R. \& Mayer, A. Enzymes of yeast polyphosphate metabolism: structure, enzymology and biological roles. Biochem Soc Trans 44, 234-239 (2016).

10. Kornberg, A., Rao, N.N. \& Ault-Riche, D. Inorganic polyphosphate: a molecule of many functions. Annu Rev Biochem 68, 89-125 (1999).

11. Auesukaree, C. et al. Intracellular phosphate serves as a signal for the regulation of the PHO pathway in Saccharomyces cerevisiae. J Biol Chem 279, 17289-17294 (2004).

12. Hothorn, M. et al. Catalytic core of a membrane-associated eukaryotic polyphosphate polymerase. Science 324, 513-516 (2009).

13. Gerasimaite, R., Sharma, S., Desfougeres, Y., Schmidt, A. \& Mayer, A. Coupled synthesis and translocation restrains polyphosphate to acidocalcisome-like vacuoles and prevents its toxicity. J Cell Sci 127, 5093-5104 (2014).

14. Denoncourt, A. \& Downey, M. Model systems for studying polyphosphate biology: a focus on microorganisms. Curr Genet (2021).

15. Uttenweiler, A., Schwarz, H., Neumann, H. \& Mayer, A. The vacuolar transporter chaperone (VTC) complex is required for microautophagy. Mol Biol Cell 18, 166-175 (2007).

16. Muller, O. et al. The Vtc proteins in vacuole fusion: coupling NSF activity to V(0) transcomplex formation. EMBO J 21, 259-269 (2002).

17. Muller, O., Neumann, H., Bayer, M.J. \& Mayer, A. Role of the Vtc proteins in V-ATPase stability and membrane trafficking. J Cell Sci 116, 1107-1115 (2003).

18. Eskes, E., Deprez, M.A., Wilms, T.\& Winderickx, J. pH homeostasis in yeast; the phosphate perspective. Curr Genet 64, 155-161 (2018).

19. Nguyen, T.Q., Dziuba, N. \& Lindahl, P.A. Isolated Saccharomyces cerevisiae vacuoles contain low-molecular-mass transition-metal polyphosphate complexes. Metallomics 11, 1298-1309 (2019).

20. Trilisenko, L. et al. The Reduced Level of Inorganic Polyphosphate Mobilizes Antioxidant and Manganese-Resistance Systems in Saccharomyces cerevisiae. Cells 8 (2019). 
21. Ryazanova, L., Zvonarev, A., Rusakova, T., Dmitriev, V. \& Kulakovskaya, T. Manganese tolerance in yeasts involves polyphosphate, magnesium, and vacuolar alterations. Folia Microbiol (Praha) 61, 311-317 (2016).

22. Desfougeres, Y., Gerasimaite, R.U., Jessen, H.J. \& Mayer, A. Vtc5, a Novel Subunit of the Vacuolar Transporter Chaperone Complex, Regulates Polyphosphate Synthesis and Phosphate Homeostasis in Yeast. J Biol Chem 291, 22262-22275 (2016).

23. Huang, D., Moffat, J. \& Andrews, B. Dissection of a complex phenotype by functional genomics reveals roles for the yeast cyclin-dependent protein kinase Pho85 in stress adaptation and cell integrity. Mol Cell Biol 22, 5076-5088 (2002).

24. Ogawa, N., DeRisi, J. \& Brown, P.O. New components of a system for phosphate accumulation and polyphosphate metabolism in Saccharomyces cerevisiae revealed by genomic expression analysis. Mol Biol Cell 11, 4309-4321 (2000).

25. Gerasimaite, R. et al. Inositol Pyrophosphate Specificity of the SPX-Dependent Polyphosphate Polymerase VTC. ACS Chem Biol 12, 648-653 (2017).

26. Wild, R. et al. Control of eukaryotic phosphate homeostasis by inositol polyphosphate sensor domains. Science 352, 986-990 (2016).

27. Azevedo, C., Livermore, T. \& Saiardi, A. Protein polyphosphorylation of lysine residues by inorganic polyphosphate. Mol Cell 58, 71-82 (2015).

28. Lonetti, A. et al. Identification of an evolutionarily conserved family of inorganic polyphosphate endopolyphosphatases. J Biol Chem 286, 31966-31974 (2011).

29. Beltrao, P. et al. Systematic functional prioritization of protein posttranslational modifications. Cell 150, 413-425 (2012).

30. Holt, L.J. et al. Global analysis of Cdk1 substrate phosphorylation sites provides insights into evolution. Science 325, 1682-1686 (2009).

31. Albuquerque, C.P. et al. A multidimensional chromatography technology for in-depth phosphoproteome analysis. Mol Cell Proteomics 7, 1389-1396 (2008).

32. Feyder, S., De Craene, J.O., Bar, S., Bertazzi, D.L. \& Friant, S. Membrane trafficking in the yeast Saccharomyces cerevisiae model. Int J Mol Sci 16, 1509-1525 (2015).

33. Ammann, S. et al. Mutations in AP3D1 associated with immunodeficiency and seizures define a new type of Hermansky-Pudlak syndrome. Blood 127, 997-1006 (2016).

34. El-Chemaly, S. \& Young, L.R. Hermansky-Pudlak Syndrome. Clin Chest Med 37, 505511 (2016).

35. Huizing, M. et al. Hermansky-Pudlak syndrome: Mutation update. Hum Mutat 41, 543580 (2020).

36. Ruiz, F.A., Lea, C.R., Oldfield, E. \& Docampo, R. Human platelet dense granules contain polyphosphate and are similar to acidocalcisomes of bacteria and unicellular eukaryotes. J Biol Chem 279, 44250-44257 (2004).

37. Puy, C. et al. Factor XII promotes blood coagulation independent of factor XI in the presence of long-chain polyphosphates. J Thromb Haemost 11, 1341-1352 (2013).

38. Choi, S.H., Smith, S.A. \& Morrissey, J.H. Polyphosphate is a cofactor for the activation of factor XI by thrombin. Blood 118, 6963-6970 (2011).

39. Choi, S.H., Smith, S.A. \& Morrissey, J.H. Polyphosphate accelerates factor V activation by factor XIa. Thromb Haemost 113, 599-604 (2015).

40. Smith, S.A. et al. Polyphosphate modulates blood coagulation and fibrinolysis. Proc Natl Acad Sci U S A 103, 903-908 (2006). 
41. Muller, F. et al. Platelet polyphosphates are proinflammatory and procoagulant mediators in vivo. Cell 139, 1143-1156 (2009).

42. Odorizzi, G., Cowles, C.R. \& Emr, S.D. The AP-3 complex: a coat of many colours. Trends Cell Biol 8, 282-288 (1998).

43. Cowles, C.R., Odorizzi, G., Payne, G.S. \& Emr, S.D. The AP-3 adaptor complex is essential for cargo-selective transport to the yeast vacuole. Cell 91, 109-118 (1997).

44. Azevedo, C. \& Saiardi, A. The new world of inorganic polyphosphates. Biochem Soc Trans 44, 13-17 (2016).

45. Bentley-DeSousa, A. \& Downey, M. From underlying chemistry to therapeutic potential: open questions in the new field of lysine polyphosphorylation. Curr Genet 65, 57-64 (2019).

46. McCarthy, L. et al. Proteins required for vacuolar function are targets of lysine polyphosphorylation in yeast. FEBS Lett 594, 21-30 (2020).

47. Bentley-DeSousa, A. et al. A Screen for Candidate Targets of Lysine Polyphosphorylation Uncovers a Conserved Network Implicated in Ribosome Biogenesis. Cell Rep 22, 3427-3439 (2018).

48. Stepp, J.D., Huang, K. \& Lemmon, S.K. The yeast adaptor protein complex, AP-3, is essential for the efficient delivery of alkaline phosphatase by the alternate pathway to the vacuole. J Cell Biol 139, 1761-1774 (1997).

49. Ruotolo, R., Marchini, G. \& Ottonello, S. Membrane transporters and protein traffic networks differentially affecting metal tolerance: a genomic phenotyping study in yeast. Genome Biol 9, R67 (2008).

50. Kapitzky, L. et al. Cross-species chemogenomic profiling reveals evolutionarily conserved drug mode of action. Mol Syst Biol 6, 451 (2010).

51. Angers, C.G. \& Merz, A.J. HOPS interacts with Ap15 at the vacuole membrane and is required for consumption of AP-3 transport vesicles. Mol Biol Cell 20, 4563-4574 (2009).

52. Rehling, P., Darsow, T., Katzmann, D.J. \& Emr, S.D. Formation of AP-3 transport intermediates requires Vps41 function. Nat Cell Biol 1, 346-353 (1999).

53. Chalfie, M., Tu, Y., Euskirchen, G., Ward, W.W. \& Prasher, D.C. Green fluorescent protein as a marker for gene expression. Science 263, 802-805 (1994).

54. Li, M., Rong, Y., Chuang, Y.S., Peng, D. \& Emr, S.D. Ubiquitin-dependent lysosomal membrane protein sorting and degradation. Mol Cell 57, 467-478 (2015).

55. Pokrzywa, W., Guerriat, B., Dodzian, J. \& Morsomme, P. Dual sorting of the Saccharomyces cerevisiae vacuolar protein Sna4p. Eukaryot Cell 8, 278-286 (2009).

56. Reggiori, F. \& Klionsky, D.J. Autophagic processes in yeast: mechanism, machinery and regulation. Genetics 194, 341-361 (2013).

57. Henne, W.M., Buchkovich, N.J. \& Emr, S.D. The ESCRT pathway. Dev Cell 21, 77-91 (2011).

58. Schmidt, O. \& Teis, D. The ESCRT machinery. Curr Biol 22, R116-120 (2012).

59. Hecht, K.A., O'Donnell, A.F. \& Brodsky, J.L. The proteolytic landscape of the yeast vacuole. Cell Logist 4, e28023 (2014).

60. Freimoser, F.M., Hurlimann, H.C., Jakob, C.A., Werner, T.P. \& Amrhein, N. Systematic screening of polyphosphate (poly P) levels in yeast mutant cells reveals strong interdependence with primary metabolism. Genome Biol 7, R109 (2006). 
61. Park, S.Y. \& Guo, X. Adaptor protein complexes and intracellular transport. Biosci Rep 34 (2014).

62. Mechler, B., Muller, M., Muller, H., Meussdoerffer, F. \& Wolf, D.H. In vivo biosynthesis of the vacuolar proteinases A and B in the yeast Saccharomyces cerevisiae. $J$ Biol Chem 257, 11203-11206 (1982).

63. Distel, B., Al, E.J., Tabak, H.F. \& Jones, E.W. Synthesis and maturation of the yeast vacuolar enzymes carboxypeptidase Y and aminopeptidase I. Biochim Biophys Acta 741, 128-135 (1983).

64. Oughtred, R. et al. The BioGRID interaction database: 2019 update. Nucleic Acids Res 47, D529-D541 (2019).

65. Stringer, D.K. \& Piper, R.C. A single ubiquitin is sufficient for cargo protein entry into MVBs in the absence of ESCRT ubiquitination. J Cell Biol 192, 229-242 (2011).

66. Zhu, L., Jorgensen, J.R., Li, M., Chuang, Y.S. \& Emr, S.D. ESCRTs function directly on the lysosome membrane to downregulate ubiquitinated lysosomal membrane proteins. Elife 6 (2017).

67. McNatt, M.W., McKittrick, I., West, M. \& Odorizzi, G. Direct binding to Rsp5 mediates ubiquitin-independent sorting of Sna3 via the multivesicular body pathway. Mol Biol Cell 18, 697-706 (2007).

68. Llinares, E., Barry, A.O. \& Andre, B. The AP-3 adaptor complex mediates sorting of yeast and mammalian PQ-loop-family basic amino acid transporters to the vacuolar/lysosomal membrane. Sci Rep 5, 16665 (2015).

69. Bru, S., Jimenez, J., Canadell, D., Arino, J. \& Clotet, J. Improvement of biochemical methods of polyP quantification. Microb Cell 4, 6-15 (2016). 


\section{FIGURE LEGENDS:}

Figure 1: GFP-Vtc5 is localized to the vacuole membrane via the conserved AP-3 pathway. A) Simplified schematic of protein transport to the vacuole in S. cerevisiae. B) The indicated strains were grown in YPD prior to incubation with FM 4-64, which marks the vacuole membrane, for 2 hours. Cells were then washed with fresh YPD for 30 minutes, transferred to synthetic media, and imaged. Live-cell fluorescence microscopy was performed using a Leica DMI 6000 microscope at 63X with oil immersion. For AP-3 mutants, green (GFP-Vtc5) images were taken at longer exposure times to account for differences in signal intensity. Images were processed in FIJI. Scale bar represents $10 \mu \mathrm{m}$. C) The indicated strains were processed as in B.

\section{Figure 2: Partial disruption of GFP-Vtc3 and GFP-Vtc4 localization in AP-3 mutants}

A) The indicated strains were grown in YPD media prior to incubation with FM 4-64, which marks the vacuole membrane, for 2 hours. Cells were then washed with fresh YPD for 30 minutes, transferred to synthetic media, and imaged. Live-cell fluorescence microscopy was performed using a Zeiss AxioObserver 7 at $63 \mathrm{X}$ with oil immersion. Images were processed in FIJI. B) The indicated strains were processed as in A. Scale bar represents $10 \mu \mathrm{m}$.

Figure 3: AP-3 mutation causes degradation of GFP-Vtc proteins. A) Upon AP-3 mutation (apl5s and aps $3 \Delta$ ), full-length GFP-Vtc5 protein levels are reduced with a concomitant increase in free GFP. Proteins were extracted from the indicated strains using a TCA protein extraction protocol, separated on a 10\% BioRad TGX Stain-Free ${ }^{\mathrm{TM}}$ FastCast $^{\mathrm{TM}}$ acrylamide gel, and transferred to a nitrocellulose membrane. The membrane was imaged on a BioRad ChemiDoc after immunoblotting with anti-GFP. Total protein was imaged as a loading control. B-C) 
Quantification of free GFP/GFP-Vtc5 ratios and GFP-Vtc5 full length levels. Quantifications were done using BioRad ImageLab software and graphs were created using Prism GraphPad Software. One-way ANOVAs were performed with Tukey posthoc tests. ${ }^{*} p<0.05, * * p<0.01$, *** $p<0.001$. Error bars represent standard deviation of the mean. $\mathrm{n}=3$ for WT and $\mathrm{n}=6$ for AP-3 mutants. D)-F) In AP-3 mutants, GFP-Vtc4-expressing strains accumulate free GFP at the expense of full-length protein. Methodology was as in A-C. G)-I) In AP-3 mutants, GFP-Vtc3expressing strains accumulate free GFP at the expense of full-length protein. The same methodology was used as in 4A-C.

\section{Figure 4: Degradation of mislocalized GFP-Vtc5 depends on ESCRT and the Pep4}

protease. A) The mislocalization of GFP-Vtc5 upon AP-3 mutation is mediated by the ESCRT pathway. Cells were grown in YPD prior to incubation with FM4-64, which marks the vacuole membrane, for 2 hours. Cells were then washed with fresh YPD for 30 minutes, transferred to synthetic media, and imaged. Live-cell fluorescence microscopy was performed using a Leica DMI 6000 at $63 \mathrm{X}$ with oil immersion. Images were processed in FIJI. Scale bar represents 10 $\mu \mathrm{m}$. White arrows indicate potential MVBs at vacuole. B) Free GFP accumulation in GFP-Vtc5, mediated by AP-3 mutation, is reversed by ESCRT ( vps $27 \Delta$ ) and pep $4 \Delta$ mutation. Proteins from the indicated strains were extracted using a TCA protein extraction protocol, separated on a $10 \%$ BioRad TGX Stain-Free ${ }^{\mathrm{TM}}$ FastCast $^{\mathrm{TM}}$ acrylamide gel, and transferred to a nitrocellulose membrane. The membrane was imaged for total protein and probed using an anti-GFP antibody to detect GFP-Vtc5 protein using a BioRad ChemiDoc. C) GFP-Vtc5 degradation and free GFP accumulation is reversed by $D O A 4$ deletion. The same methodology was used as in $4 \mathrm{~B}$. D) 
Deletion of DOA4 rescues localization of GFP-Vtc5 in apl5 $\triangle$ mutants. The same methodology was used as in $4 \mathrm{~A}$.

Figure 5: Functional AP-3 is required for the maintenance of polyP levels. A) AP-3 subunit mutant causes a reduction in polyP levels. There is a loss of polyP when mutating each of the

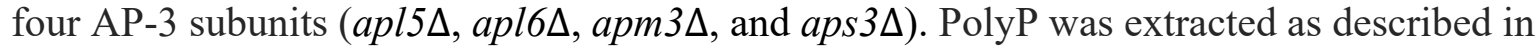
Experimental Procedures. After extraction, samples were mixed with polyP sample buffer and separated on a $15.8 \%$ TBE-Urea Acrylamide gel. The gel was incubated in fixing solution with toluidine blue for 15 minutes prior to destaining and imaging. PolyP standards $(14,60$, and 120 Pi units) are included as standards. B) AP-3 mutants (apl5 $\Delta$ and $a p s 3 \Delta$ ) cause a reduction in polyP levels when GFP-Vtc5 is expressed under a GPD1 promoter. The same methodology was used as in 5A. C) Mutating both AP-3 (apl5s) and $v t c 5 \Delta$ results in a largely epistatic decrease in polyP levels. The same methodology was used as in 5A. D) Although doa4t is capable of rescuing GFP-Vtc5 localization and protein degradation when AP-3 is mutated (apl5A), it is incapable of restoring polyP levels. The same methodology was used as in 5A. Asterisk indicates a background contaminant in extractions.

Figure 6: GFP-Vtc5 is localized to the vacuole membrane via the AP-3 complex. In the absence of AP-3, mislocalized GFP-Vtc5 could be localized at the plasma membrane or elsewhere in the cell prior to recognition by the ESCRT complex which delivers it to the vacuole lumen for Pep4-dependent degradation. However, we cannot rule out the possibility that Vtc5 is incorrectly inserted at the vacuole membrane prior to ESCRT-dependent internalization. See text for details. 


\section{Supplementary Figure 1: Polyphosphorylation of Apl5 does not impact protein transport.}

A) The AP-3 complex is a conserved heterotetramer which includes two large subunits (Ap15/AP3 $\beta 1$ and Ap16/AP381), one medium subunit (Apm3/AP3 $\mu 1)$, and one small subunit (Aps3/AP3 61$)$. B) Mutating lysines to arginines (K-R) within Ap15's PASK cluster causes a collapse in the electrophoretic shift on NuPAGE, indicating a loss of polyphosphorylation. Proteins from the indicated strains were extracted using a TCA protein extraction protocol, electrophoresed on a 4-12\% NuPAGE gel, and transferred to a PVDF membrane. The membrane was developed with autoradiography film after immunoblotting with an anti-GFP antibody to detect C-terminal Ap15-GFP fusion alleles. Anti-Rts1 serves as a positive control for polyphoshorylation. Ponceau S stain is used as a loading control. C) GFP-Vtc5 is localized to the vacuole membrane in Ap15PASKK-R mutants. Cells were grown in YPD prior to incubation with FM 4-64, which marks the vacuole membrane, for 2 hours. Cells were then washed with fresh YPD for 30 minutes, transferred to synthetic media, and imaged. Live-cell fluorescence microscopy was performed using a Leica DMI 6000 microscope at $63 \mathrm{X}$ with oil immersion. In AP-3 null mutant (apl5 $\Delta)$, green (GFP-Vtc5) images were taken at longer exposure times to account for differences in signal intensity. Images were processed in FIJI. Scale bar represents 10

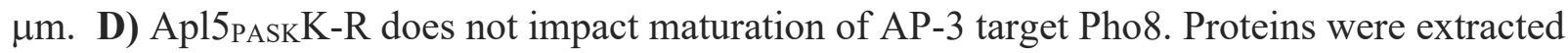
from the indicated strains using a TCA protein extraction protocol, electrophoresed on a $12 \%$ SDS-PAGE gel, and transferred to a PVDF membrane. The membrane was developed with autoradiography film after immunoblotting with an anti-Pho8 antibody. Ponceau S stain is used as a loading control. 


\section{Supplemental Figure 2: Deletion of the Apl5 PASK cluster does not impact sensitivity to} rapamycin or nickel chloride. A) Functional AP-3 is required for growth in the presence of $0.003 \mu \mathrm{M}$ rapamycin. Cells were grown in YPD to log phase and diluted to $0.1 \mathrm{OD}_{600}$ prior to 5fold serial dilutions in $\mathrm{H}_{2} 0.4 \mu \mathrm{L}$ of each dilution was spotted on the indicated media prior to incubation at $30{ }^{\circ} \mathrm{C}$ for 2-4 days. Images were taken on a Bio-Rad ChemiDoc. DMSO is used as a control for rapamycin treatment. B) Functional AP-3 is required for growth in the presence of $0.75 \mathrm{mM} \mathrm{NiCl}_{2}$. The same methodology was used as described in Supp. 2A with YPD plates used as a control.

\section{Supplemental Figure 3: ESCRT complex is responsible for GFP-Vtc5 degradation only}

when AP-3 is non-functional. A) In wild-type cells, GFP-Vtc5 remains localized to the vacuole

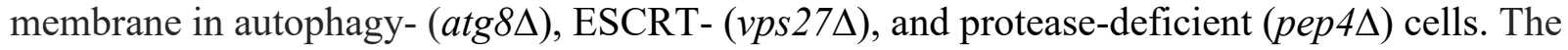
indicated strains were grown in YPD prior to incubation with FM 4-64, which marks the vacuole membrane, for 2 hours. Cells were then washed with fresh YPD for 30 minutes, transferred to synthetic media, and imaged. Live-cell fluorescence microscopy was performed using a Leica DMI 6000 microscope at $63 \mathrm{X}$ with oil immersion. Images were processed in FIJI. Scale bar

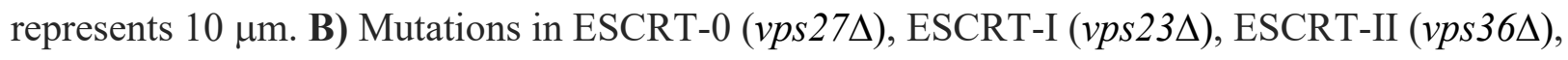
and ESCRT-III (vps204) reverse free GFP accumulation in AP-3 mutants expressing GFP-Vtc5. Proteins were extracted using a TCA protein extraction protocol, electrophoresed on a $10 \%$ BioRad TGX Stain-Free ${ }^{\mathrm{TM}}$ FastCast $^{\mathrm{TM}}$ acrylamide gel, and transferred to a nitrocellulose membrane. The membrane was imaged using a BioRad ChemiDoc after immunoblotting with an anti-GFP antibody to detect GFP-Vtc5. Total protein was imaged as a loading control. C) 
Deletion of $D O A 4$ does not impact wild-type GFP-Vtc5 localization. The same methodology was used as in Supp. 3A.

Supplemental Table 1 - Yeast strains used in this work (Excel File Tab 1)

Supplemental Table 2 - Antibodies used in this work (Excel File Tab 2) 
bioRxiv preprint doi: https://doi.org/10.1101/2021.03.15.434853; this version posted March 16, 2021. The copyright holder for this preprint (which was not certified by peer review) is the author/funder, who has granted bioRxiv a license to display the preprint in perpetuity. It is made available under aCC-BY-NC 4.0 International license.

FIGURE 1: Vtc5 is localized to the vacuole membrane via the conserved AP-3 pathway

A

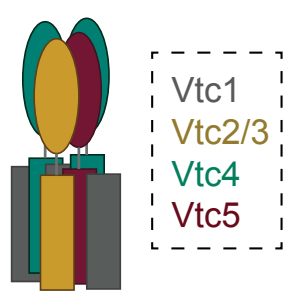

VTC Complex
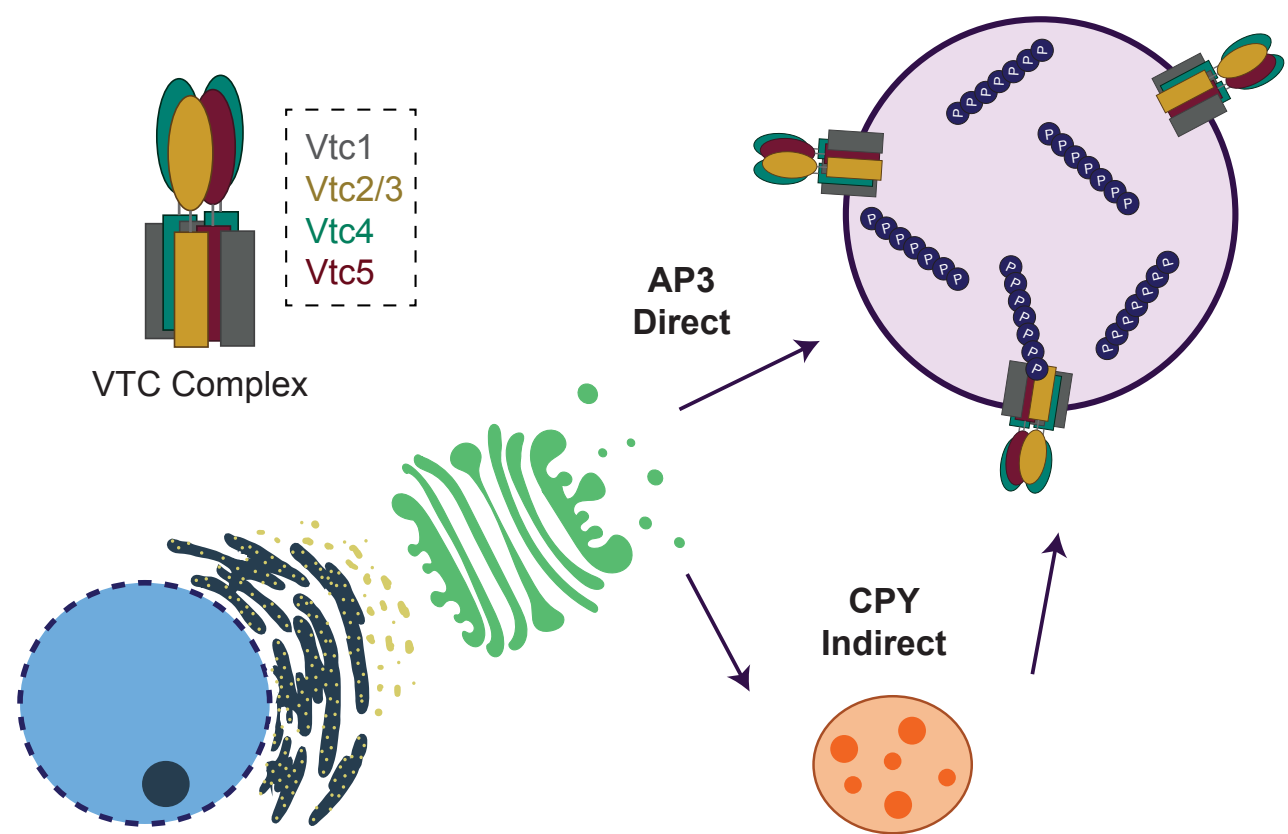

B
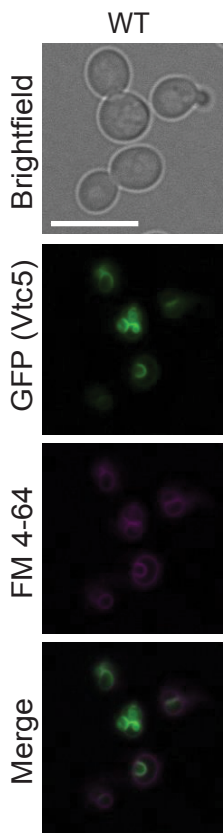

ap/5s
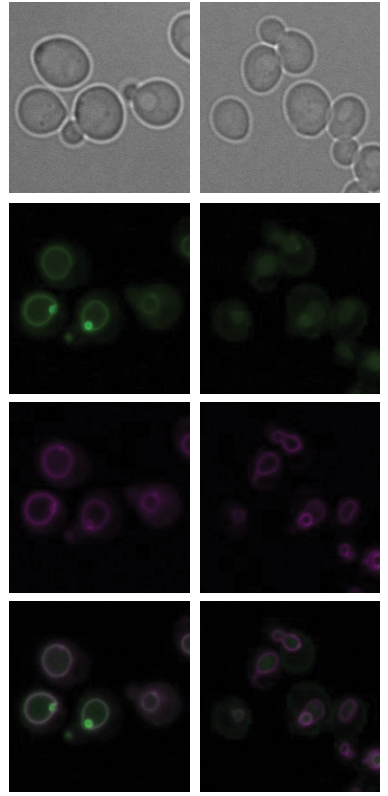
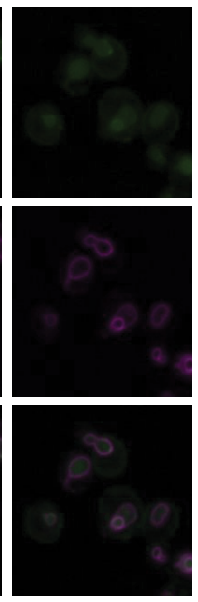

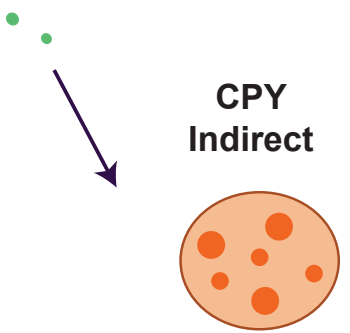

C
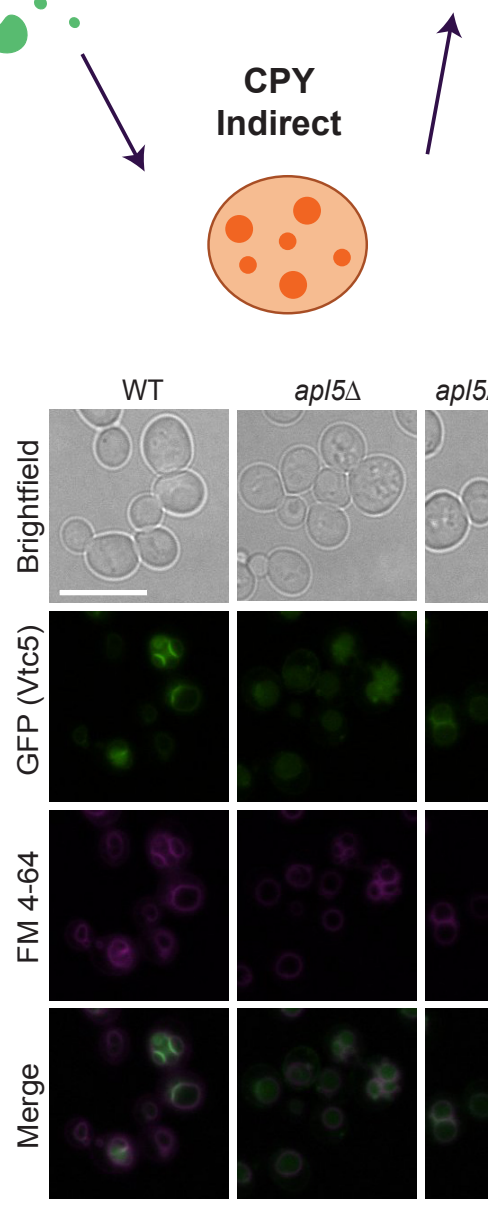

ap/5 $897-932$

$\operatorname{aps} 3 \Delta$
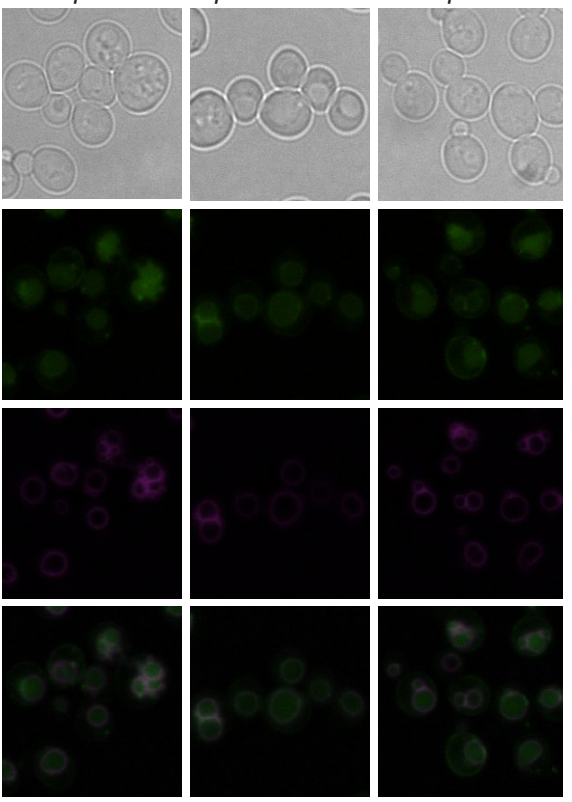
FIGURE 2: Partial disruption of Vtc3 and Vtc4 localization in AP-3 mutants

A
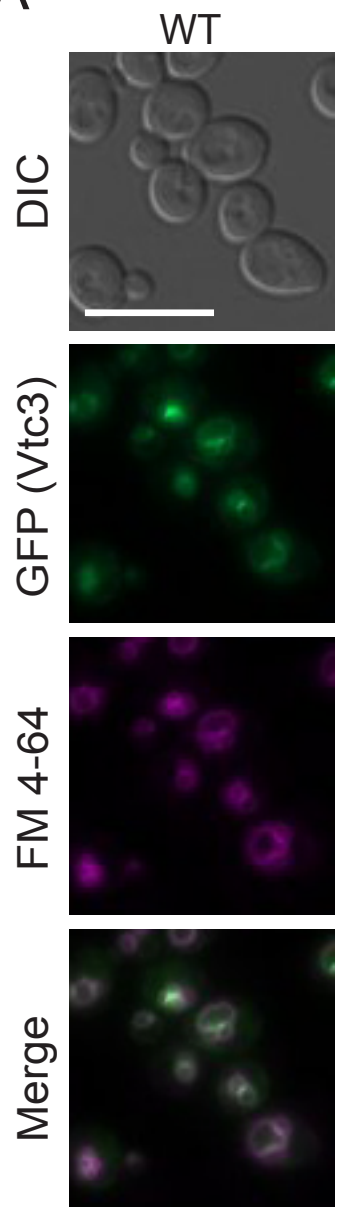

ap/5s
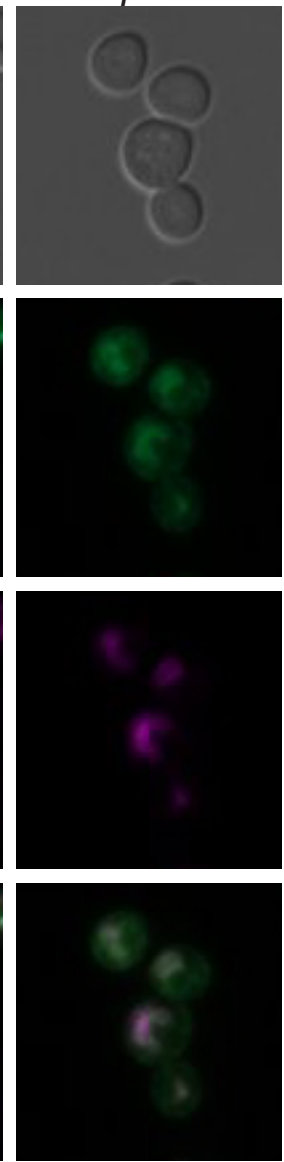

B
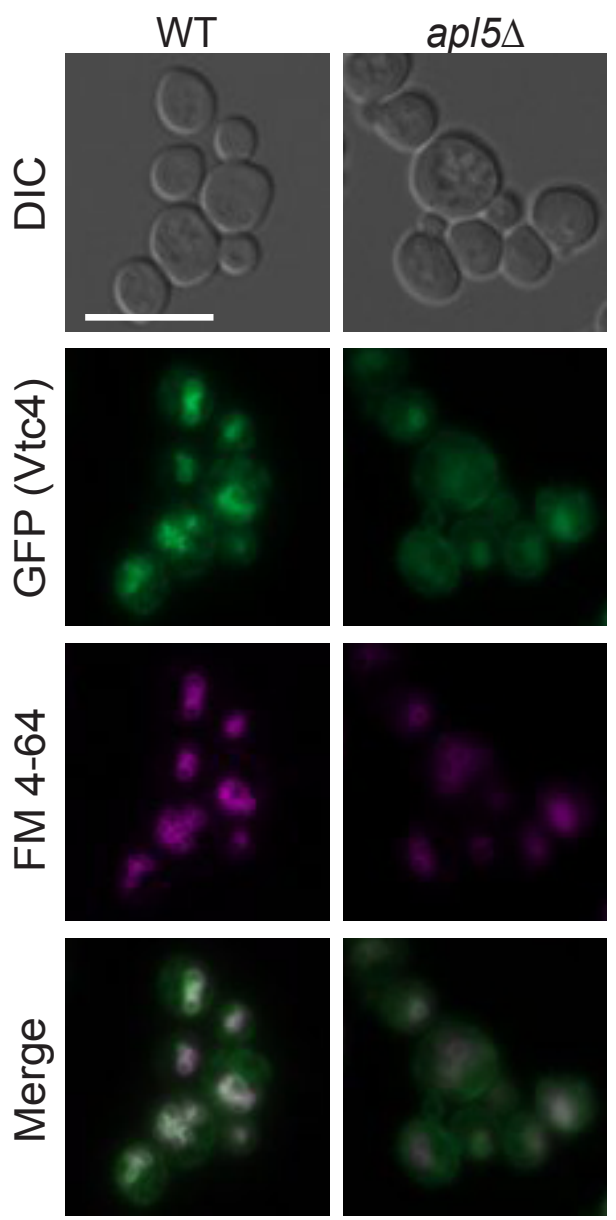
FIGURE 3: AP-3 mutation causes degradation of GFP-Vtc proteins

A

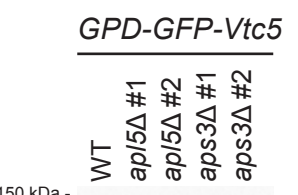

$150 \mathrm{kDa}$

$100 \mathrm{kDa}-$

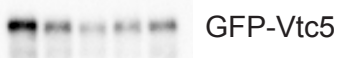

$75 \mathrm{kDa}$ -

$50 \mathrm{kDa}$ -

$37 \mathrm{kDa}-$

$25 \mathrm{kDa}-$
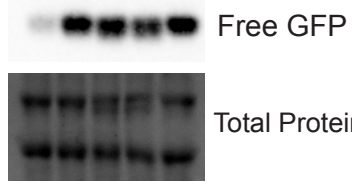

Total Protein

$\mathrm{B}$

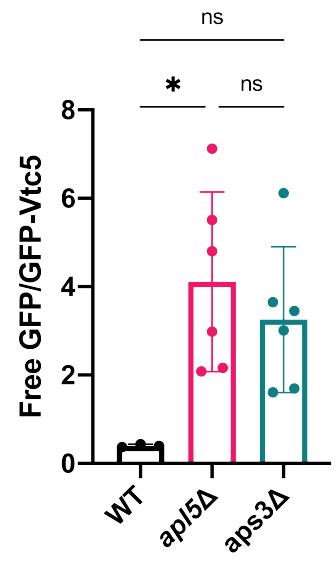

C

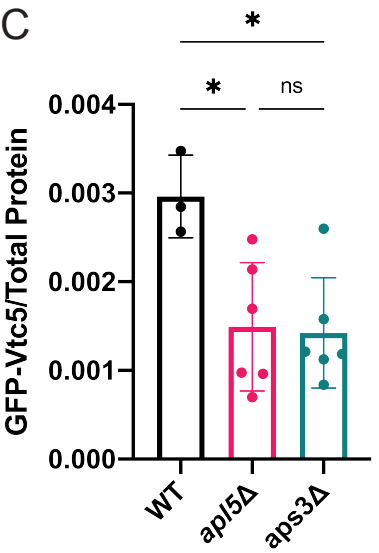

D

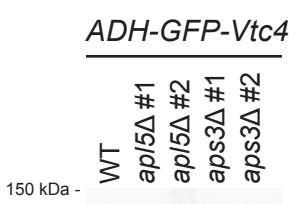

$100 \mathrm{kDa}$ -$$
\text { - }
$$

GFP-Vtc4

$75 \mathrm{kDa}$ -

$50 \mathrm{kDa}$ -

$37 \mathrm{kDa}$ -

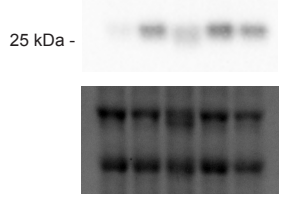

Free GFP

Total Protein

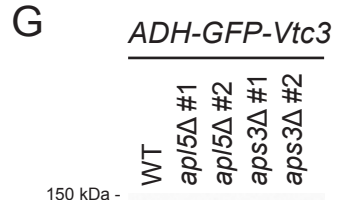

$100 \mathrm{kDa}$ -

$--\infty$ GFP-Vtc3

$75 \mathrm{kDa}$ -

$50 \mathrm{kDa}$ -

$37 \mathrm{kDa}$ -

$25 \mathrm{kDa}$ -

Free GFP

E
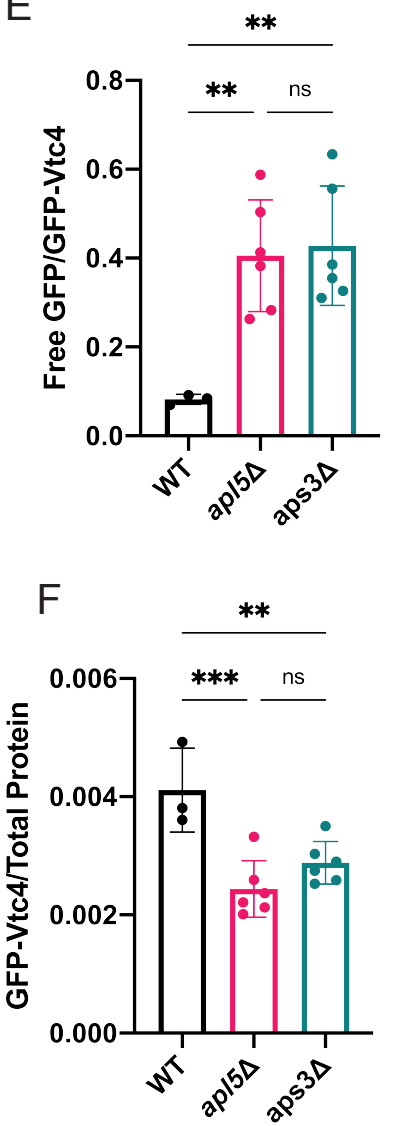
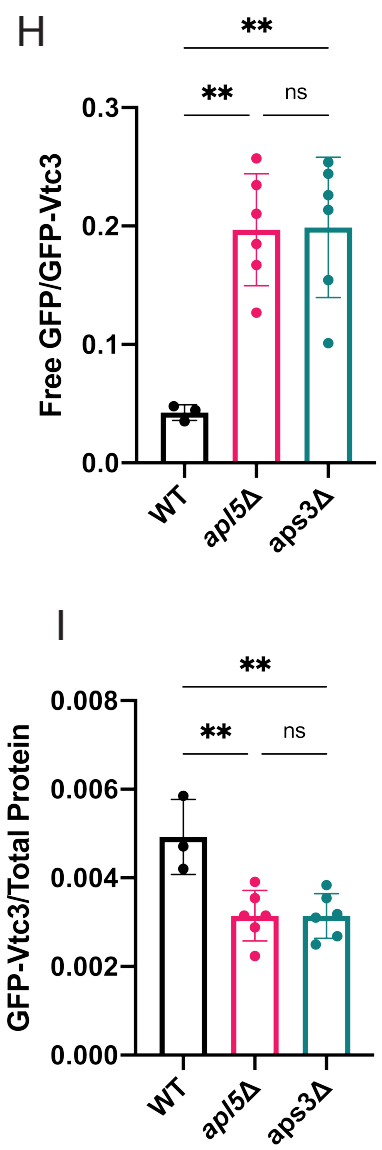
bioRxiv preprint doi: $\mathrm{https}$ //doi.org/10.1101/2021.03.15.434853; this version posted March 16, 2021. The copyright holder for this preprint (which was not certified by peer review) is the author/funder, who has granted bioRxiv a license to display the preprint in perpetuity. It is made available under aCC-BY-NC 4.0 International license.

FIGURE 4: Degradation of mislocalized Vtc5 depends on ESCRT and the Pep4 protease

A

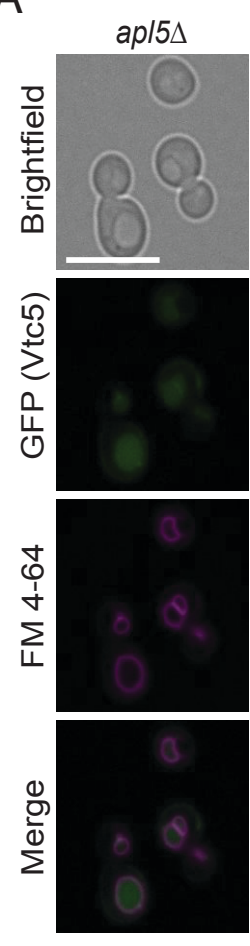

$\operatorname{ap} / 5 \Delta \operatorname{atg} 8 \Delta$

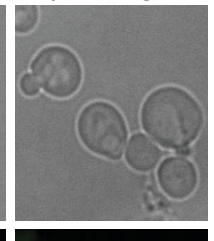

ap $/ 5 \Delta$ vps $27 \Delta$

$a p / 5 \Delta$ pep $4 \Delta$
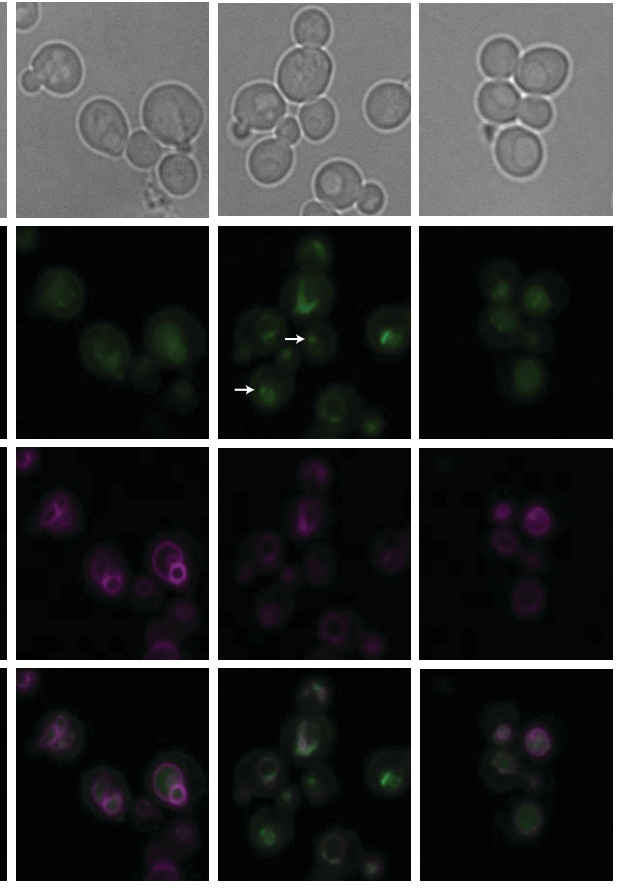

B

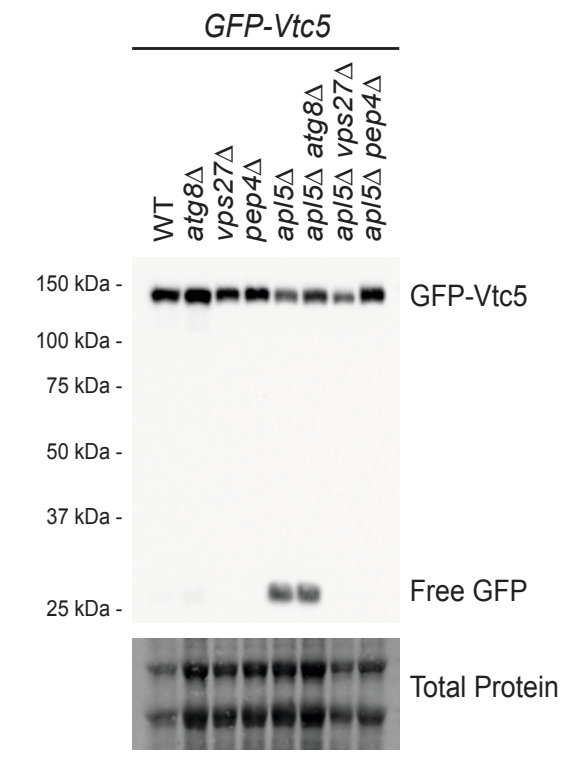

D

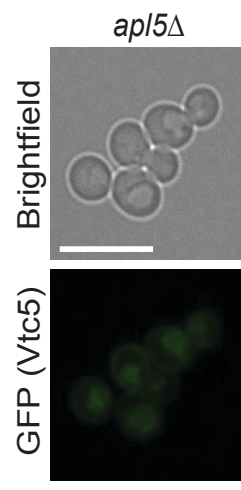

ap $/ 5 \Delta$ doa $4 \Delta$

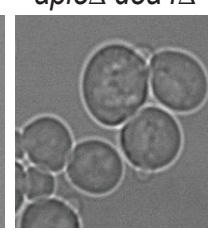

$\sum \frac{0}{2} \frac{\pi}{\frac{\pi}{5}}$

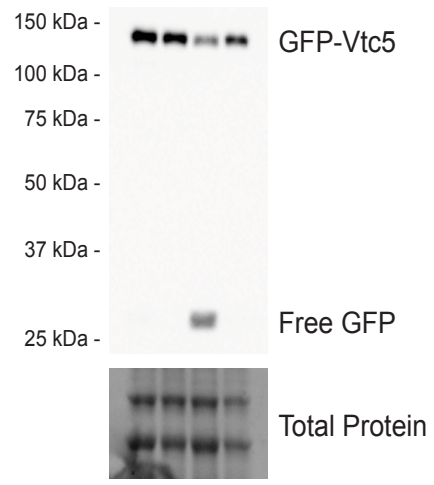

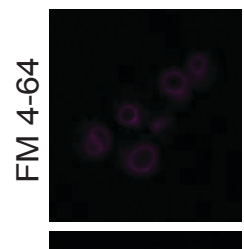
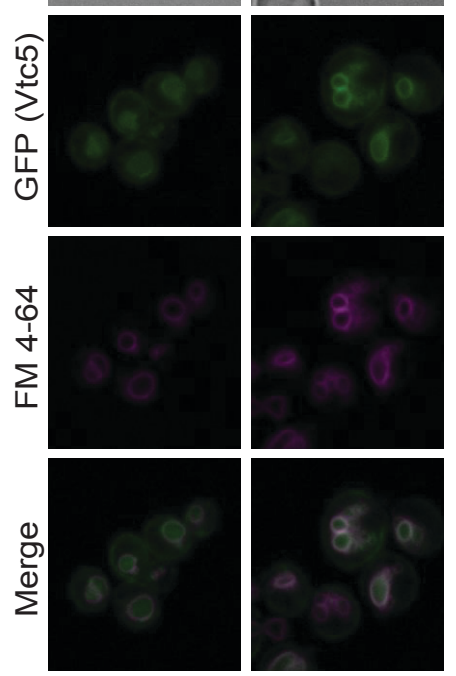
FIGURE 5: Functional AP-3 is required for the maintenance of polyP levels

A

PolyP
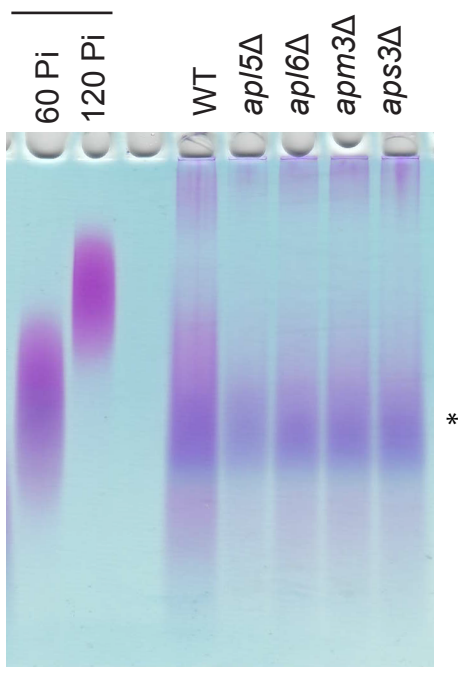

C

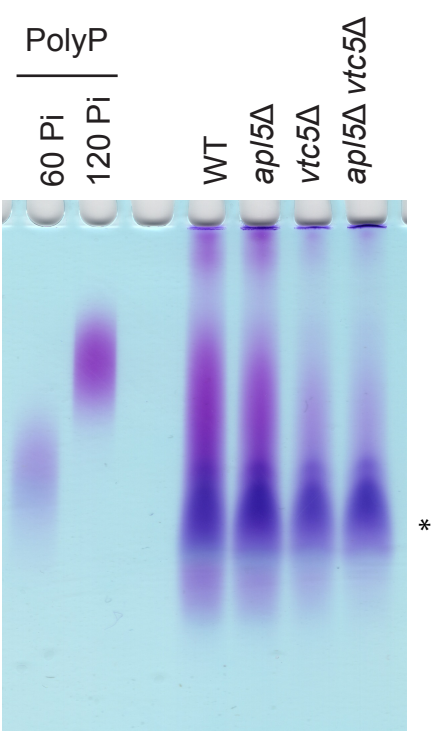

B

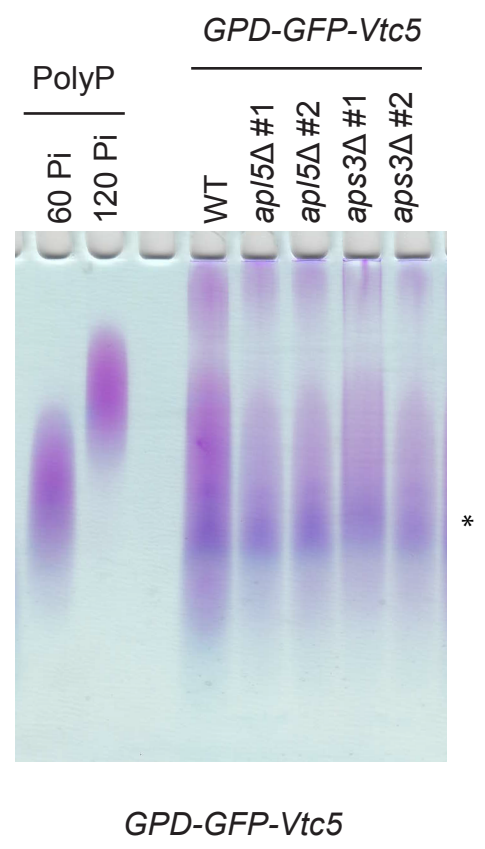


FIGURE 6: Vtc5 is localized to the vacuole membrane via the AP-3 complex
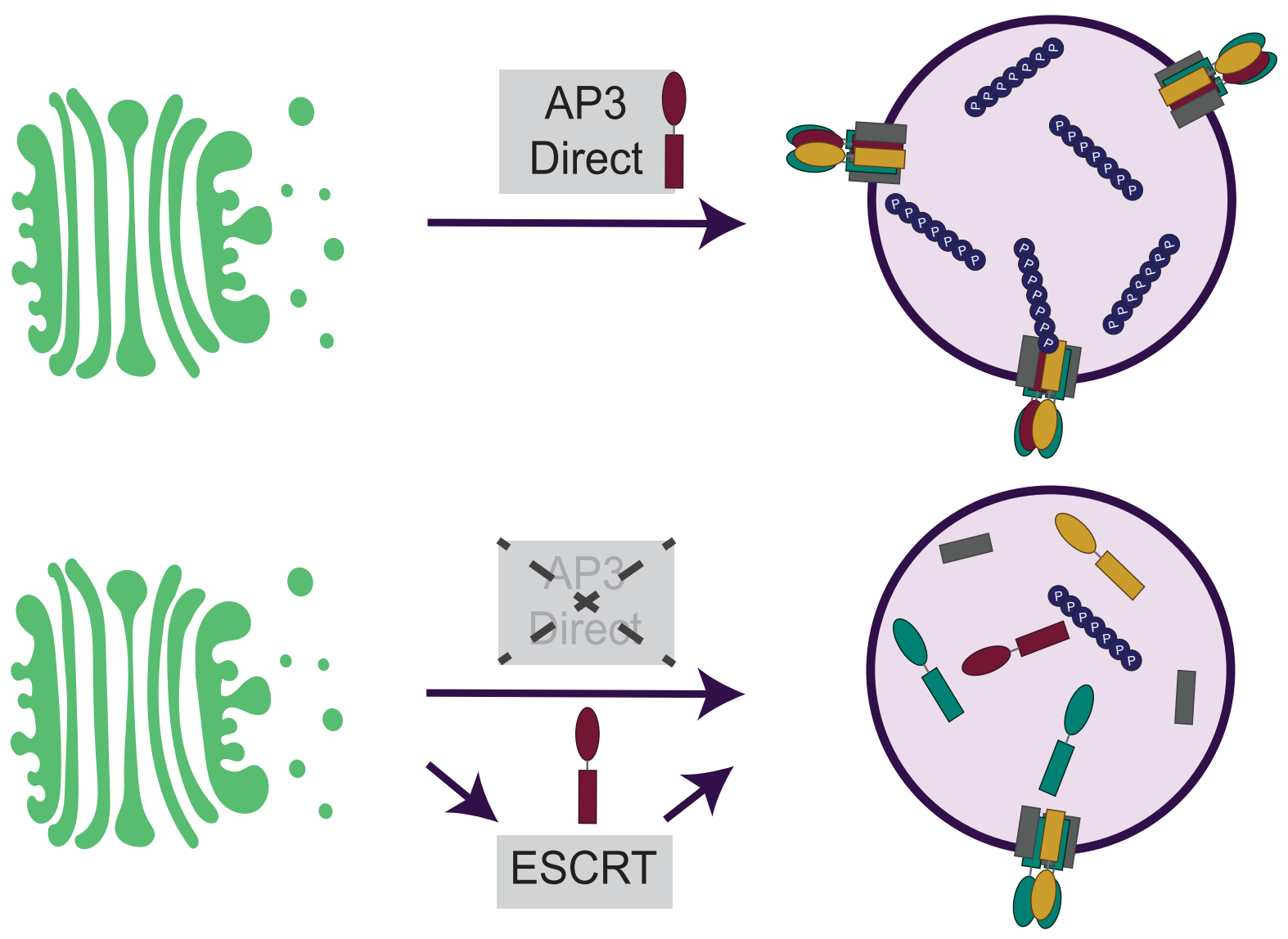
FIGURE S1: Polyphosphorylation of Apl5 does not impact protein transport

A

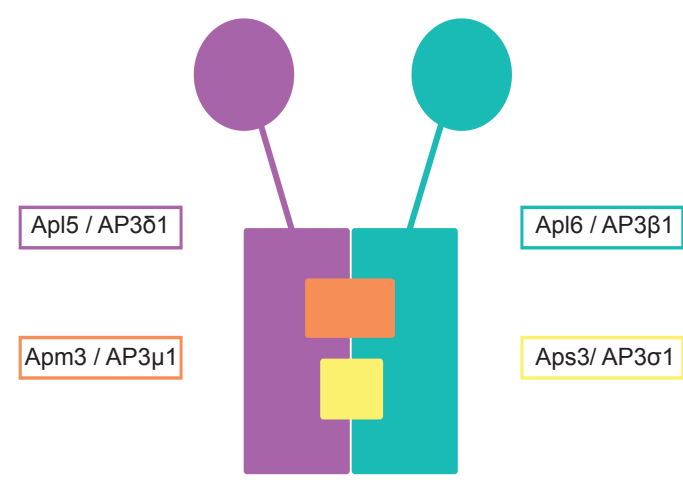

C

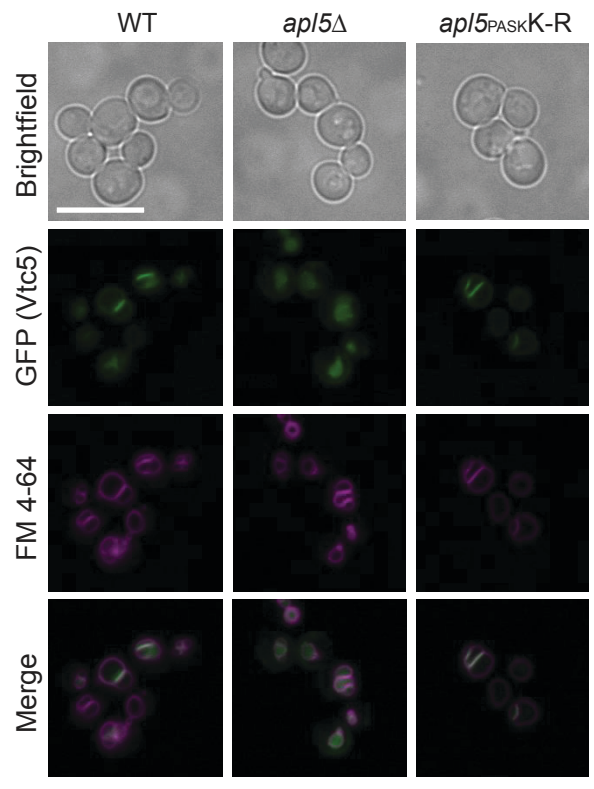

B

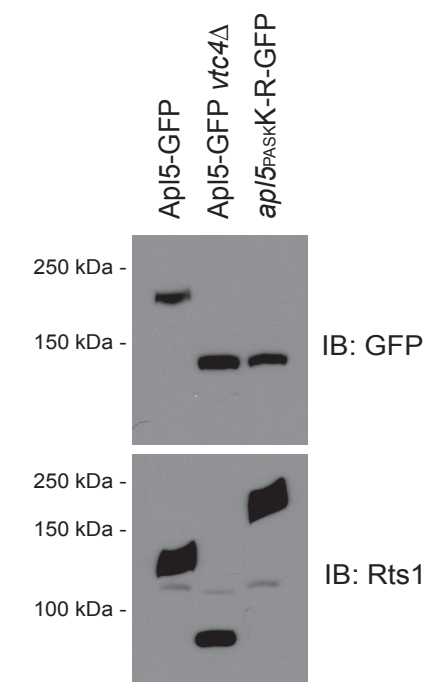

Total Protein

D

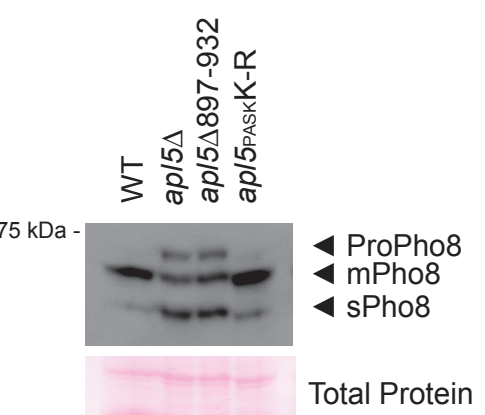

IB: Pho8 (ALP) 
FIGURE S2: Deletion of the Apl5 PASK motif does not impact sensitivity to rapamycin or nickel chloride.

A

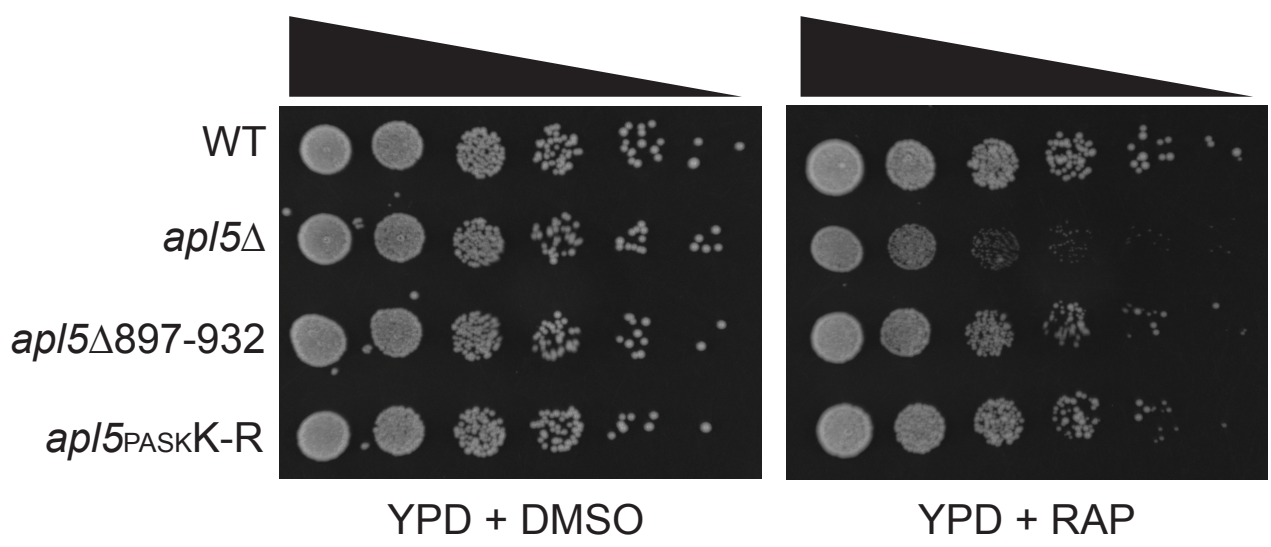

B

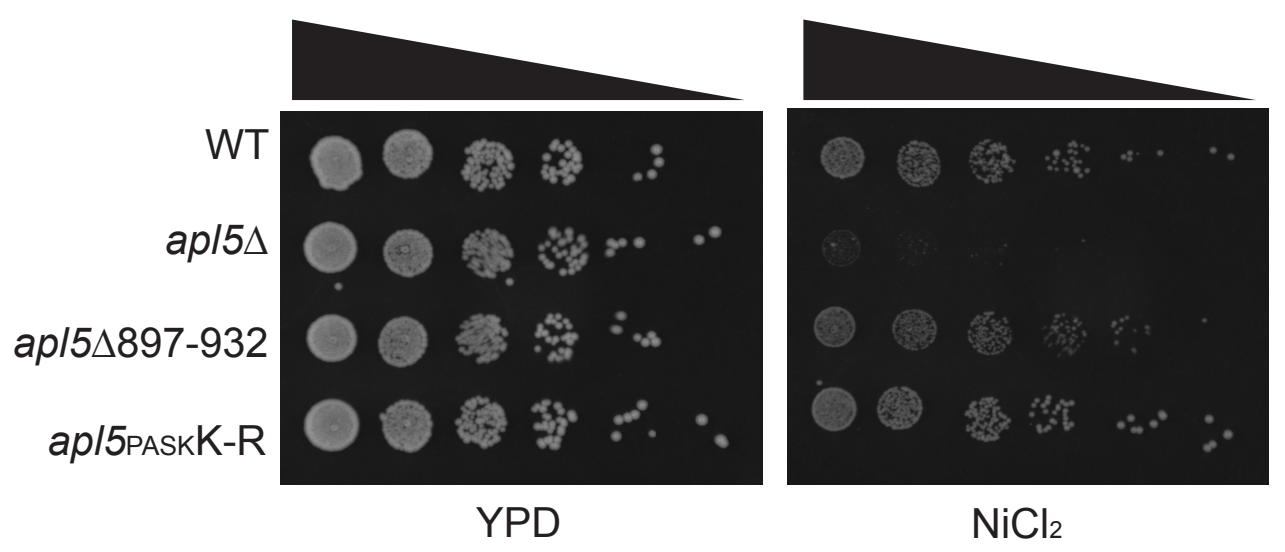


FIGURE S3: ESCRT complex is responsible for Vtc5 degradation when AP-3 is non-functional

A
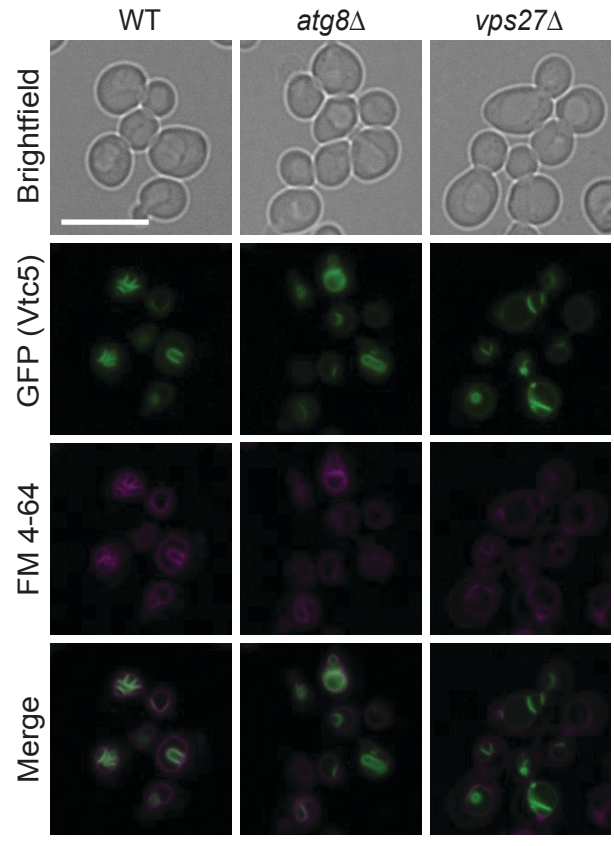

C

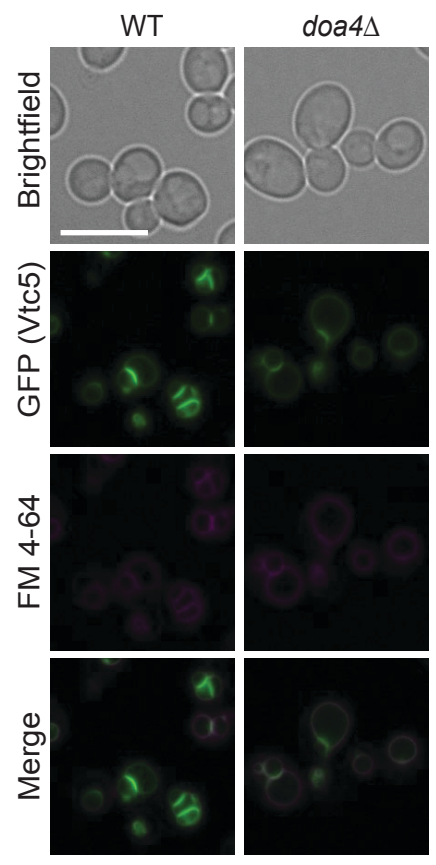

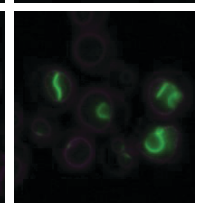
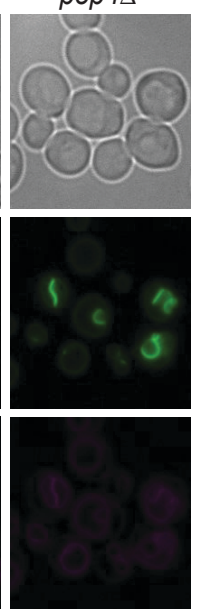

B

\begin{tabular}{|c|}
\hline GFP-Vtc5 \\
\hline 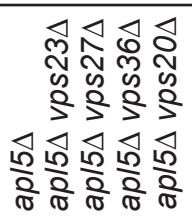 \\
\hline
\end{tabular}

150 kDa -

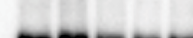

100 kDa -

75 kDa -

50 kDa -

37 kDa -

25 kDa -
Free GFP

GFP-Vtc5

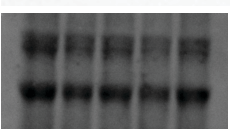

Total Protein

Free GFP 